\title{
On the onset of galactic winds in quiescent star forming galaxies
}

\author{
Y. Dubois and R. Teyssier
}

\author{
Service d'Astrophysique, CEA/DSM/DAPNIA/SAp, Centre d'Études de Saclay, L'Orme des Merisiers, \\ 91191 Gif-sur-Yvette Cedex, France \\ e-mail: ydubois@cea.fr
}

Received 20 July 2007 / Accepted 1 October 2007

\begin{abstract}
Context. The hierarchical model of galaxy formation, despite its many successes, still overpredicts the baryons fraction locked in galaxies as a condensed phase. The efficiency of supernovae feedback, proposed a long time ago as a possible solution for this socalled "overcooling" problem, is still under debate, mainly because modelling supernovae explosions within a turbulent interstellar medium, while capturing realistic large scale flows around the galaxy is a very demanding task.

Aims. Our goal is to study the effect of supernovae feedback on a disc galaxy, taking into account the impact of infalling gas on both the star formation history and the corresponding outflow structure, the apparition of a supernovae-driven wind being highly sensitive to the halo mass, the galaxy spin and the star formation efficiency.

Methods. We model our galaxies as cooling and collapsing NFW spheres. The dark matter component is modelled as a static external potential, while the baryon component is described by the Euler equations using the AMR code RAMSES. Metal-dependent cooling and supernovae-heating are also implemented using state-of-the-art recipes coming from cosmological simulations. We allow for three parameters to vary: the halo circular velocity, the spin parameter and the star formation efficiency.

Results. We found that the ram pressure of infalling material is the key factor limiting the apparition of galactic winds. We obtain a very low feedback efficiency, with supernovae to wind energy conversion factor around one percent, so that only low circular velocity galaxies give rise to strong winds. For massive galaxies, we obtain a galactic fountain, for which we discuss the observational properties.

Conclusions. We conclude that for quiescent isolated galaxies, galactic winds appear only in very low mass systems. Although this can quite efficiently enrich the IGM with metals, they do not carry away enough cold material to solve the overcooling problem.
\end{abstract}

Key words. galaxies: formation - galaxies: evolution - galaxies: structure - methods: numerical

\section{Introduction}

The hierarchical model of structure formation, for which massive galaxies grow by mergers of smaller satellites or by filamentary accretion of gas, is now a well-established theory (the so-called CDM paradigm), which compares favourably with observations. In this standard picture, dark matter is the main component that drives structure formation via gravitational instability from primordial density perturbations. On the other hand, numerical simulations including gas dynamics with radiative cooling and some recipes of star formation tend to produce galactic discs which are too small, too dense, with too low angular momentum and too many condensed baryons, when compared to observations. These various problems, termed "overcooling" (Dekel \& Silk 1986; Blanchard et al. 1992; Navarro \& White 1993; Yepes et al. 1997; Gnedin 1998; Hultman \& Pharasyn 1999; Somerville \& Primack 1999; Cole et al. 2000; Kay et al. 2002; Springel \& Hernquist 2003) or the "angular momentum" problem (Navarro \& Benz 1991; Sommer-Larsen et al. 1999; Steinmetz \& Navarro 1999; Bullock et al. 2001; Maller \& Dekel 2002; Abadi et al. 2003; Sommer-Larsen et al. 2003; Read \& Gilmore 2005), may all have the same physical origin. Nevertheless, they are considered as weak points in the CDM theory.

Supernovae (SNe)-driven winds are a key ingredient of current galaxy formation models, in order to suppress the formation of low-mass galaxies and maybe to solve the "overcooling" problem. They can potentially suppress the fast consumption of gas by transferring cold and dense gas into the hot and diffuse surrounding medium of a galaxy. Thus, a fraction of gas would not collapse into cold dense molecular clouds and would not transform into stars. This is the most favoured explanation for the disruption of star formation and the lack of baryons available in stars (Springel \& Hernquist 2003; Rasera \& Teyssier 2006; Stinson et al. 2006). Feedback is also invoked to reproduce the large disc galaxies observed at our epoch (Weil et al. 1998; Thacker \& Couchman 2001), the morphology of galactic discs (Sommer-Larsen et al. 2003; Okamoto et al. 2005) to stabilise the gaseous disc against the Toomre instability (Robertson et al. 2004) or to fit the temperature of the intergalactic medium (IGM) at redshift $\sim 3$ (Cen \& Bryan 2001).

The proper modelling of galactic winds is quite difficult to handle, especially in cosmological simulations, because of the very large scale separation between the galactic outflow in the IGM and the SNe explosions within the interstellar medium (ISM). However it is an unavoidable task to solve the weakest points of the hierarchical model, both in semi-analytical models (White \& Frenk 1991; Somerville \& Primack 1999; Kauffmann et al. 1999; Cole et al. 2000; Hatton et al. 2003; Monaco 2004; Bertone et al. 2005) and in numerical simulation (Cen \& Ostriker 1992; Navarro \& White 1993; Mihos \& Hernquist 1994; Katz et al. 1996; Thacker \& Couchman 2000; Scannapieco et al. 2001; Springel \& Hernquist 2003; Rasera \& Teyssier 2006) of galaxy formation. Observational evidence for galactic outflows has already been pointed out by several authors (Bland \& Tully 1988; 
Heckman et al. 1990; Ellison et al. 2000; Heckman et al. 2000; Pettini et al. 2001, 2002; Adelberger et al. 2003; Bouché et al. 2006). They are usually associated with massive starbursts, for which very strong outflows are reported: for one solar mass of stars formed in the galaxy, between 1 and 5 solar mass of gas are ejected in the winds of starburst dwarf galaxies (Martin 1999).

Despite the difficulties of modelling SNe explosions within a turbulent, multiphase and magnetised ISM, understanding the physics of the resulting large scale outflows is also a challenge. As explored by Fujita et al. (2004) in the context of an isolated, pre-formed galactic disc, the ram-pressure of infalling material might be the main limiting factor for galactic winds to exist. The gravitational potential is likely to play a minor role in the development of galactic winds, especially in low-mass galaxies where the escape velocity is far lower than the velocity of the wind. This fact, outlined first by Fujita et al. (2004), is in contradiction with most semi-analytical studies so far, for which only the escape velocity of the parent halo determines if a wind can develop or not. In this paper, using a self-consistent modelling of gas cooling and accretion from the hot surrounding medium into the disc, we will demonstrate that it is indeed the ram pressure of the infalling gas that matters for galactic outflows.

To illustrate further the difficulties in modelling $\mathrm{SNe}$ feedback, we note that the mass ejection rates measured in galactic winds obtained in numerical simulations are widely discrepant. Some authors report very efficient winds, even in the quite massive galaxies (Springel \& Hernquist 2003; Tasker \& Bryan 2006), while other authors obtained very low ejection efficiency, mainly in dwarf galaxies (Mac Low \& Ferrara 1999). We believe that these differences arise partly from the different numerical techniques used, and partly from the different boundary conditions imposed around the galaxy. In this paper, we report the first self-consistent adaptive mesh refinement (AMR) simulation of a collapsing NFW (Navarro et al. 1996) spherical cloud forming a galactic disc from the inside out. In the recent literature, this same problem was addressed using smooth particle hydrodynamics (SPH) techniques reported by Springel \& Hernquist (2003). Grid-based simulations were also performed, but using isolated, pre-formed rotating discs (Fujita et al. 2004; Tasker \& Bryan 2006). One of the key outcome of such numerical simulations is the fraction of the injected SNe energy which is transferred up to the large scale galactic wind and is truly available for feedback (Fujita et al. 2004; Scannapieco et al. 2006).

We are indeed facing a major difficulty when introducing feedback in numerical simulations that include radiative losses: the characteristic timescale of radiative cooling in dense regions where star formation occurs is very fast, usually faster than the simulation time step, so that one relies on implicit time integration schemes. As a consequence, if one handles SNe heating by dumping thermal energy in the same dense regions, most (if not all) of the energy is radiated away before any large scale flow develops. This spurious effect has been known for a long time, both in cosmological simulations (Katz 1992; Mihos \& Hernquist 1994; Katz et al. 1996) and in ISM simulations (Wada et al. 2000; Joung \& Mac Low 2006). We follow the recipe proposed by Mori et al. (1997) and Gnedin (1998) by adding around each SN event density, velocity and energy profiles based on the Sedov blast wave solution, to obtain the correct kinetic to thermal energy fraction in the flow. In this way, a large fraction of kinetic energy is injected into the disc, without being radiated away on the spot. This will translate into a turbulent gaseous disc, and in some cases into a large scale outflow. To account for the small scale effect of SNe feedback, we follow the multiphase approach of Yepes et al. (1997), Ascasibar et al. (2002), Springel \& Hernquist (2003), and Marri \& White (2003), for which the SNe thermal energy is collectively accounted for as a new equation of state for the dense ISM, depending on the gas local density (polytropic equation of state). In this way, we can reproduce a stiffer equation of state for the dense gas, leading to thicker, more stable discs (Toomre 1964; Robertson et al. 2004). Another problem arises because the scales where radiative losses of $\mathrm{SNe}$ remnants occur are largely unresolved $(\lesssim 1 \mathrm{pc})$. It is therefore likely that the energy transfer of SNe remnants to the ISM is much lower (Larson 1974; Thornton et al. 1998) than the energy transferred from supperbubbles initiated by multiple localised SNe (Dekel \& Silk 1986; Melioli \& de Gouveia Dal Pino 2004). In reality, modelling properly the energy deposition requires solving of the coherence length of the SNe explosions. Thus, we have to bear in mind that our supperbubble model entails many assumptions.

Within this framework, we would like to answer the following questions: what is the conversion efficiency between the small scale SNe luminosity and the large scale outflow luminosity? What are the conditions for a galactic wind to develop and escape from the parent halo potential well? What is the mass ejection rate of such a wind? What is the metallicity of the wind and other associated observational signatures?

Although the cooling NFW halo approach that we present in this paper represents an important improvement over previous isolated disc grid-based simulations, we are still a long way from realistic cosmological simulations. Such idealised simulations are nevertheless useful to understand the physical processes responsible for the apparition of a galactic wind subject to boundary conditions which are relevant for cosmology. They also give us the possibility of deriving a simple analytical model which highlights the importance of infall in the process of wind formation. Our paper is organised as follows. In Sect. 2 we present our analytical model and introduce the relevant quantities for constraining the galactic wind formation epoch. In Sect. 3, we present our simulation settings, with the physics involved in our different disc simulations, as well as some numerical details. In Sect. 4, we present our main result, namely the formation of either a galactic outflow or a galactic fountain. We compare the actual wind formation epoch with our analytical prediction. We also describe in details the flow structure in each case, and discuss its observational signature.

\section{Galactic winds from cooling NFW halos}

In this section, we describe the simplified problem we would like to study using high-resolution numerical simulations, as well as a simple analytical model that guides us in interpreting the results. We model an isolated NFW halo (Navarro et al. 1996), with initially similar distribution for the gas and the dark matter components (Ascasibar et al. 2003). We follow the initial settings proposed by Springel \& Hernquist (2003), so our work can be directly compared to them. Imposing identical profiles for the gas and the dark matter is especially important in the outer part, to have realistic accretion rates at late times, when the outer region of the halo is falling into the inner disc. Both density profiles are therefore given by

$\rho=\frac{\rho_{\mathrm{s}}}{r / r_{\mathrm{s}}\left(1+r / r_{\mathrm{s}}\right)^{2}}$,

so that the total integrated mass is

$M(<r)=4 \pi \rho_{\mathrm{s}} r_{\mathrm{s}}^{3}\left(\ln \left(1+r / r_{\mathrm{s}}\right)-\frac{r / r_{\mathrm{s}}}{1+r / r_{\mathrm{s}}}\right)$, 
$\rho_{\mathrm{S}}$ is the characteristic density of the halo and $r_{\mathrm{s}}$ its characteristic radius. We define here the Virial radius as the radius where the average density is equal to 200 times the critical density, assuming $H_{0}=70 \mathrm{~km} \mathrm{~s}^{-1} \mathrm{Mpc}^{-1}$. We consider a constant gas fraction of $15 \%$ throughout the halo. The temperature profile is adjusted so that a strict hydrostatic equilibrium of the gas sphere is maintained in the non-cooling, non-rotating case. The halo is truncated at 2 Virial radii: outside the halo, we imposed a constant gas density equal to a small fraction $\left(10^{-4}\right)$ of the density at this radius. Note that this truncation radius is a key parameter in this study. The size of the box is taken to be equal to 6 Virial radius. The concentration parameter $c=r_{\mathrm{vir}} / r_{\mathrm{s}}$ of the NFW profile is taken to be 10 , independent of the halo mass. Initially, our halo is slowly rotating, with an angular momentum profile corresponding to the average profile found in 3D cosmological simulations (Bullock et al. 2001), for which the specific angular momentum is

$j(r)=j_{\max } \frac{M(<r)}{M_{\mathrm{vir}}}$

with a spin parameter $\lambda=J|E|^{1 / 2} /\left(G M_{\mathrm{vir}}^{5 / 2}\right)$, for which we consider only two representative values $\lambda=0.04$ and 0.1 . Starting from this initial equilibrium configuration, the gas is allowed to radiate its thermal energy using standard, metal-dependant cooling processes. Note that initially we assume a constant metallicity equal to $10^{-3}$ solar throughout the halo. In agreement with the standard picture of galaxy formation, a centrifugally supported disc quickly forms in the halo centre, gradually growing from the inside out. Its size depends strongly on the chosen spin parameter. When enough dense material is formed within the disc, star formation is allowed to proceed in a quiescent way, using a star formation recipe based on a standard Schmidt law (see Eq. (10)). According to Salpeter (1955) IMF, for each solar mass of stars formed, $\eta_{\mathrm{SN}} \simeq 0.1$ solar masses are recycled into $\mathrm{SNe}$ ejecta, and drive strong shock waves into the surrounding ISM material.

Our goal is to implement these two rather standard physical ingredients in the RAMSES code, to study the formation of galactic winds. This will be described in the next section. In the present section, we develop a simple analytical model which allows us to capture the basic mechanism driving galactic winds. Our picture contrasts with that discussed in Fujita et al. (2004), who assumed a pre-formed gaseous galactic disc, with no selfconsistent star formation. A blast wave was initiated at the very centre of the disc, with an energy budget consistent with massive star bursts. Depending on the blast wave and disc parameters, two possible scenarios emerge: "blow out" for which the disc is completely destroyed, and "blow away", corresponding to a galactic wind escaping the galactic disc. These authors have demonstrated that the main limiting factor for a wind to appear in the "blow away" case is the amount of infalling gas, coming from the surrounding halo. Here we address this question using a different approach, based on quiescent star formation and infall from a cooling NFW halo.

Let us consider that the gas is cooling instantaneously, so that pressure support is lost from the very beginning. Each spherical shell is therefore free-falling, gradually accelerating towards the centre. We compute the accretion rate of halo material onto the disc using a standard semi-analytical recipe (e.g. Hatton et al. 2003), namely by taking the time derivative of the integrated accreted mass

$M_{\text {acc }}(<t)=M\left(<r_{\mathrm{ff}}(t)\right)$,

where $r_{\mathrm{ff}}(t)$ is the radius for which the free-fall time $t_{\mathrm{ff}}(r)$ is equal to the current time $t$. The gaseous disc mass therefore grows by accretion and decays because of star formation, leading to a simple differential equation for its time evolution

$\dot{M}_{\mathrm{g}}=\dot{M}_{\mathrm{acc}}-\frac{M_{\mathrm{g}}}{\left\langle t_{*}\right\rangle}$.

Here, $\left\langle t_{*}\right\rangle$ is the (mass-weighted) average star formation rate (SFR) within the disc. It depends on the exact disc geometry (size and thickness) and on the Schmidt law parameters. As a first order approximation, one can show that

$\left\langle t_{*}\right\rangle \propto t_{0} \lambda_{0}$

small discs form stars more efficiently than large ones. If one assumes that $\left\langle t_{*}\right\rangle$ remains constant in time, one gets the following formal solution

$M_{\mathrm{g}}(t)=\int_{0}^{t} \dot{M}_{\mathrm{acc}}(u) \exp \left(\frac{u-t}{\left\langle t_{*}\right\rangle}\right) \mathrm{d} u$.

We finally deduce the total SNe luminosity in the disc using

$L_{\mathrm{SN}}=\eta_{\mathrm{SN}} \epsilon_{\mathrm{SN}} \dot{M}_{*}=\eta_{\mathrm{SN}} \epsilon_{\mathrm{SN}} \frac{M_{\mathrm{g}}}{\langle t\rangle_{*}}$

where $\epsilon_{\mathrm{SN}} \simeq 50 \mathrm{keV}$ is the specific energy produced by one single SN $\left(10^{51}\right.$ erg for an average progenitor mass of $\left.10 M_{\odot}\right)$. Part of the SNe energy contributes to sustaining a turbulent multiphase ISM. Only a (small) fraction of this energy is converted into actual wind luminosity escaping from the dense gaseous disc. The fraction of energy that manage to escape from the dense gaseous disc will depend on the disc characteristic (thickness, size, gas content). In our simulations, these properties will be specified by the the spin parameter of the halo and by the star formation timescale of the Schmidt law. We parametrise this unknown conversion factor $\chi$ by defining the wind luminosity as $L_{\mathrm{W}}=\chi L_{\mathrm{SN}}$. Our goal is to perform numerical simulations of star forming discs in order to compute the actual conversion efficiency resulting from various, complex hydrodynamical processes within the disc. We thus call this parameter $\chi$ the hydrodynamical efficiency.

Our goal is now to derive conditions under which a galactic wind can form within the halo. We know from previous studies (Fujita et al. 2004) that, in presence of infalling gas, the main factor preventing the wind from blowing out is the ram pressure of the surrounding material. This is a much more stringent constraint than any criterion based on the escape velocity. If we assume that the free-falling cold gas hits the disc plane with terminal velocity, we can compute the accretion luminosity as

$L_{\mathrm{acc}}(t)=\frac{1}{2} V_{0}^{2}\left(r_{\mathrm{ff}}(t)\right) \dot{M}_{\mathrm{acc}}$ and $V_{0}^{2}(r)=-2 \int_{0}^{r} \frac{G M(x)}{x^{2}} \mathrm{~d} x$.

We define the wind beak-out epoch $t_{\mathrm{W}}$ as the time for which the wind luminosity exceeds the accretion luminosity $L_{\mathrm{acc}}\left(t_{\mathrm{W}}\right)=$ $L_{\mathrm{W}}\left(t_{\mathrm{W}}\right)$. In Fig. 1, the wind break-out epoch is shown for various halo masses, as a function of our unknown parameter $\chi$. One clearly sees that the smaller the hydrodynamical efficiency, the later the wind will blow out of the disc. More importantly, for each halo mass, there is a minimum efficiency below which no wind can form at all. This minimal value for $\chi$ is around $0.7 \%$ (resp. $3 \%$ and $15 \%$ ) for a $10^{10} M_{\odot}$ halo (resp. $10^{11} M_{\odot}$ and $10^{12} M_{\odot}$ ). We conclude from this very simple model that it is critical to determine the actual hydrodynamical efficiency in quiescent star forming galaxies. Our goal here is to perform high resolution numerical simulations of isolated galaxies, to measure the epoch when galactic winds appear and deduce from $t_{\mathrm{W}}$ the corresponding (most likely) hydrodynamical efficiency. 


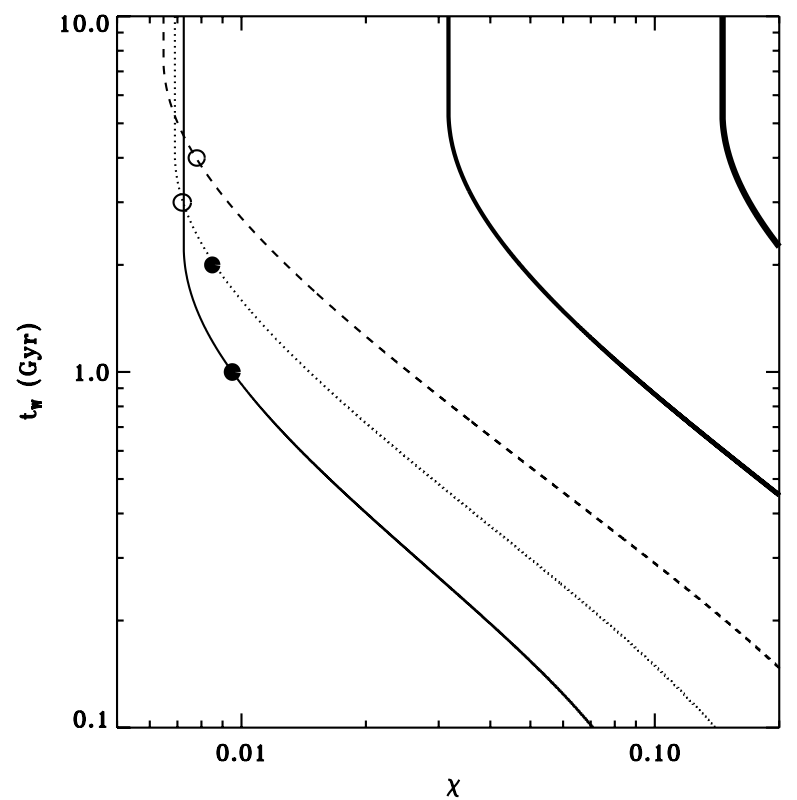

Fig. 1. The epoch of wind formation $t_{\mathrm{W}}$ in Gyr as a function of the hydrodynamical efficiency $\chi$. We have assumed three different average star formation times $\left\langle t_{*}\right\rangle=1 \mathrm{Gyr}$ (thin solid line), $\left\langle t_{*}\right\rangle=2 \mathrm{Gyr}$ (dotted line) and $\left\langle t_{*}\right\rangle=4 \mathrm{Gyr}$ (dashed line) for the $10^{10} M_{\odot}$ halo, and the same $\left\langle t_{*}\right\rangle=3 \mathrm{Gyr}$ for the $10^{11} M_{\odot}$ (solid line) and the $10^{12} M_{\odot}$ (thick solid line) halos. Open circles are for Sc $\left(t_{\mathrm{W}}=3 \mathrm{Gyr}\right)$ and $\mathrm{Sd}\left(t_{\mathrm{W}}=4 \mathrm{Gyr}\right)$, circles are for $\mathrm{Sa}\left(t_{\mathrm{W}}=1 \mathrm{Gyr}\right)$ and $\mathrm{Sb}\left(t_{\mathrm{W}}=2 \mathrm{Gyr}\right)$.

\section{Numerical methods}

Our simulations were performed with the AMR code RAMSES (Teyssier 2002). The gas evolution is computed using a secondorder Godunov scheme for the Euler equations, while collisionless star particles' trajectories are computed using a ParticleMesh solver. The dark matter component is accounted for as a constant background gravitational potential. Gas cooling is taken into account as a source term in the energy equation. The cooling function is computing using the Sutherland \& Dopita (1993) cooling model, using a look-up table in the temperature and metallicity plane. Metals created in the surrounding of $\mathrm{SNe}$ explosions are advected by the hydrodynamics solver as a passive scalar.

We now describe in more detail our three main physical ingredients used for the present study, namely star formation, SNe thermal feedback and SNe kinetic feedback.

\subsection{Star formation recipe}

In each cell, gas is converted into star particles following a Schmidt law

$\dot{\rho}_{*}=-\frac{\rho}{t_{*}}$ if $\rho>\rho_{0}, \quad \dot{\rho}_{*}=0$ otherwise,

where $\rho_{0}$ is an arbitrary density threshold defining what we consider here as being "ISM". The star formation timescale is proportional to the local free-fall time,

$t_{*}=t_{0}\left(\frac{\rho}{\rho_{0}}\right)^{-1 / 2}$.

The two parameters $\rho_{0}$ and $t_{0}$ are poorly known and scale dependent. One standard approach consists of calibrating these numbers to the observed SFR in local galaxies, the so-called Kennicutt (1998) law, which translates roughly into $\rho_{0} \simeq 0.1 \mathrm{H} \mathrm{cm}^{-3}$ and $t_{0} \simeq 1-10 \mathrm{Gyr}$. In the present study, we allow $t_{0}$ to take two values, namely 3 or 8 Gyr corresponding to star formation efficiencies of $\sim 0.05$ and 0.02 respectively. The latter value is compatible with Krumholz \& Tan (2007) if we suppose that star formation efficiency remains the same at very high $\left(>10^{5} \mathrm{H} \mathrm{cm}^{-3}\right)$ and unresolved densities (maximum density in our simulations stalls to a few $10^{1} \mathrm{H} \mathrm{cm}^{-3}$ and $10^{2} \mathrm{H} \mathrm{cm}^{-3} \mathrm{re}-$ spectively for the $10^{10} M_{\odot}$ and $10^{11} M_{\odot}$ due to the polytropic equation of state). When a gas cell is eligible for star formation, $N$ collisionless star particles are spawned using a Poisson random process, with probability

$P(N)=\frac{\lambda_{P}}{N !} \exp \left(-\lambda_{P}\right)$,

where the mean value is

$\lambda_{P}=\left(\frac{\rho \Delta x^{3}}{m_{*}}\right) \frac{\Delta t}{t_{*}}$.

The mass of a star particle is taken to be an integer multiple of $m_{*}=\rho_{0} \Delta x_{\min }^{3} /\left(1+\eta_{\mathrm{SN}}+\eta_{\mathrm{W}}\right)$ (see Appendix A for details on $\left.\eta_{\mathrm{W}}\right)$, and is therefore directly connected to the code spatial resolution (Rasera \& Teyssier 2006). The minimum mass of a single star particle is therefore $\sim 500 M_{\odot}$, but high density regions can spawn more massive star particles. We also ensure that no more than $90 \%$ of the gas in a cell is depleted by the star formation process.

\subsection{Polytropic equation of state}

The second important ingredient for our galactic disc simulations is the thermal feedback of SNe into the ISM, based on the multiphase model of Ascasibar et al. (2002) and Springel \& Hernquist (2003). The idea is to assume that the ISM is driven by small scale effects (turbulence, thermal instability, thermal conduction, molecular cloud formation and evaporation) which quickly reach steady-state and lead to a quasi-equilibrium thermal state, for which the average temperature is a function of the mass density alone. In practice, when the gas density exceeds the ISM threshold $\rho_{0}$, the gas temperature is forced to be equal to or higher than

$T=T_{0}\left(\frac{\rho}{\rho_{0}}\right)^{\gamma_{0}-1}$

where $\gamma_{0}$ is the polytropic index, and can be chosen to the exact value of the multiphase model (Springel \& Hernquist 2003) or to a constant value for the sake of simplicity. If $\gamma_{0}$ is chosen between $4 / 3$ and 2, as prescribed by Springel \& Hernquist (2003), the resulting equation of state gives similar results for the disc thickness and maximum density. Our standard choice is here $\gamma_{0}=5 / 3$.

\subsection{Blast wave model}

The main problem of the previous thermodynamical approach is that feedback is only accounted for at mesoscopic scales. On the other hand, it is now an observational fact that very large SNedriven bubbles appear quite naturally in star forming galaxies. These superbubbles are built by the coalescence of numerous SNe remnants, who collectively create these large-scale features, driving very large, macroscopic flows which eventually give rise to galactic winds or galactic fountains. Current cosmological 
simulations do not have the necessary resolution to resolve individual $\mathrm{SNe}$ blast wave. On the other hand, they do have the resolution to resolve superbubbles. Our goal is to inject kinetic energy in the form of spherical blast waves of size comparable to galactic superbubbles, namely $r_{\mathrm{SN}}=100$ to $200 \mathrm{pc}$. This kinetic energy injection can be considered as a turbulent forcing term with injection scale $r_{\mathrm{SN}}$.

It is now well-established that $\mathrm{SNe}$ energy cannot be released only by direct thermal energy injection (Navarro \& White 1993). For high density star forming regions, the gas radiates away all this thermal energy in one time-step due to very fast atomic cooling. The consequence is that $\mathrm{SNe}$ have no effect on the dynamics. Various methods have been proposed to correctly incorporate the SNII kinetic feedback into numerical simulations. The first idea, discussed for example in Governato et al. (2006) is to artificially stop radiative cooling in the region where the SNII explosion occurs, for a time long enough for the blast wave to develop and expand. This trick allows for a large enough fraction of the SNII energy to be converted into actual gas kinetic energy. The second approach is to directly inject kinetic energy into the surrounding gas. Springel \& Hernquist (2003) used for this purpose high-velocity collisionless particles which travel over a rather large distance (up to a few $\mathrm{kpc}$ ) before being re-incoporated to the fluid as new SPH particles.

In this paper, we directly add to each flow variable (density, momentum and total energy) a spherical blast wave solution of radius $r_{\mathrm{SN}}=150 \mathrm{pc}$, assuming that each $\mathrm{SNe}$ releases half of its energy in this kinetic form (the other half is accounted for in the polytropic equation of state). As soon as this distance is large enough compared to the grid resolution, we minimise spurious energy losses. Each time a "star" particle is created, we simultaneously remove an equivalent gas mass from the cell that is brought about by the SNII blast wave. Recalling that $m_{*}$ is the mass locked into long-lived stars, the total mass removed from each star forming cell is $m_{*}\left(1+\eta_{\mathrm{SN}}+\eta_{\mathrm{W}}\right)$, where the driving parameter $\eta_{\mathrm{W}}$ is defined as in Springel \& Hernquist (2003). We have used $\eta_{\mathrm{W}} \simeq 1$ in order to reproduce roughly a Sedov blast wave solution in typical galactic discs for which the density is slightly greater than $\rho_{0}$. Note that in order to implement this numerical scheme, we have used "debris" particles to describe the Sedov blast wave profile around each SNII explosion. Details are given in the Appendix. The most important parameter is $r_{\mathrm{SN}}$, the radius of the spherical blast wave. This sets the injection scale of the turbulent cascade in the disc (Joung \& Mac Low 2006). This value, comparable to the typical size of galactic superbubbles (McKee \& Ostriker 1977), is however larger than individual e remnants, whose size just before the snow plow phase varies from 10 to $50 \mathrm{pc}$, depending on the exact early shock wave dynamics, whether adiabatic or evaporative (Cioffi et al. 1988). We have not tried to study the impact of this important parameter on our results, to avoid clouding the purpose of this paper. We prefer to keep this parameter fixed to its theoretical value of $r_{\mathrm{SN}}=150 \mathrm{pc}$ (McKee \& Ostriker 1977; Shull \& Silk 1979).

\subsection{Simulation parameters}

We use for the fluid solver simple "outflow" boundary conditions, for which all flow variable are assumed to have zero gradient at the boundary. All simulations are initialised with a NFW gas and dark matter halo in hydrostatic equilibrium, with the same density profile for both fluids. The dark matter component is not explicitly simulated here; only baryons (stars and gas) are self-gravitating. We therefore add a fixed analytical gravitational potential to the solution of the Poisson equation.
Table 1. Parameters used for the simulations performed in this paper.

\begin{tabular}{ccccccc}
\hline \hline Run & $\begin{array}{c}V_{\text {vir }} \\
\mathrm{km} \mathrm{s}^{-1}\end{array}$ & $\lambda$ & $\begin{array}{c}t_{0} \\
\mathrm{Gyr}\end{array}$ & $\ell_{\min }$ & $\ell_{\max }$ & $\begin{array}{c}\Delta x \\
\mathrm{pc}\end{array}$ \\
\hline $\mathrm{Sa}$ & 35 & 0.04 & 3 & 7 & 11 & 73 \\
$\mathrm{Sb}$ & 35 & 0.04 & 8 & 7 & 11 & 73 \\
$\mathrm{Sc}$ & 35 & 0.1 & 3 & 7 & 11 & 73 \\
$\mathrm{Sd}$ & 35 & 0.1 & 8 & 7 & 11 & 73 \\
$\mathrm{La}$ & 75 & 0.04 & 3 & 7 & 12 & 78 \\
$\mathrm{Lb}$ & 75 & 0.04 & 8 & 7 & 12 & 78 \\
$\mathrm{Lc}$ & 75 & 0.1 & 3 & 7 & 12 & 78 \\
$\mathrm{Ld}$ & 75 & 0.1 & 8 & 7 & 12 & 78 \\
\hline
\end{tabular}

We have performed our simulations for two different halo circular velocities, namely $V_{\text {vir }}=35$ and $75 \mathrm{~km} \mathrm{~s}^{-1}$, which correspond to Virial masses of $10^{10} M_{\odot}$ and $10^{11} M_{\odot}$ at redshift zero. We have explored two different star formation efficiencies, with timescales $t_{0}=3 \mathrm{Gyr}$ and $t_{0}=8 \mathrm{Gyr}$. We use a coarse grid of $128^{3}$ cells, to properly describe the large scale dynamics within the halo, which corresponds, in the RAMSES terminology, to a minimum level of refinement $\ell_{\min }=7$. The grid is further refined up to 4 or 5 additional levels of refinement $\left(\ell_{\max }=11\right.$ or 12 corresponding to $\sim 75 \mathrm{pc}$, see Table 1 for more details), based on a quasi-Lagrangian strategy, for which a cell is refined if its mass exceeds a threshold of $2 \times 10^{-6}$ the Virial mass. This refinement strategy verifies the Jeans length criterion inside the galactic disc because of the polytropic component which helps to stabilise against fragmentation. Starting with $2 \times 10^{6}$ cells, our simulations usually reach a total of 4 to $5 \times 10^{6}$ cells. We end our simulation after a typical integration time of $6 \mathrm{Gyr}$, which corresponds to the free-fall time of the halo outer boundary at 2 Virial radius.

\section{Simulation results}

In this section, we present our simulation results for the two different halo mass considered in this paper, namely $10^{10}$ and $10^{11} M_{\odot}$. In the first case, a strong wind generally develops after a few Gyr. We will examine in detail the wind structure and physical properties. For the second case, because of the ram pressure of infalling gas from the surrounding halo, we see no wind forming: a clear galactic fountain sets in and creates a turbulent, hot atmosphere above (and below) the rotating disc. We will study the structure of this galactic fountain, and compare the various density and velocity profiles obtained in both cases (wind versus fountain).

\section{1. $10^{10} M_{\odot}$ halo}

In Fig. 4, we have plotted the SFR obtained in the low mass galaxies ( $\mathrm{Sa}, \mathrm{Sb}, \mathrm{Sc}$ and $\mathrm{Sd}$, see Table 1 for details). The smallest disc with the highest star formation efficiency (run Sa) shows, as expected, the highest peak in the SFR. Using our analytical model, we are in a position to roughly recover the same curve using $t_{*} \simeq 1 \mathrm{Gyr}$.

Run $\mathrm{Sb}$ and $\mathrm{Sc}$ have roughly the same value for the product $t_{0} \lambda_{0}$, and therefore, as predicted by our simple analytical model (Eqs. (6) and (7)), have roughly the same star formation history $\left(t_{*} \simeq 2 \mathrm{Gyr}\right)$. The largest disc with the lowest star formation efficiency $\left(t_{*} \simeq 4 \mathrm{Gyr}\right)$ has the lowest SFR. At late time, after 3 to 4 Gyr, the 4 curves converge to roughly the same value. This corresponds to the late epoch when star formation is mainly 

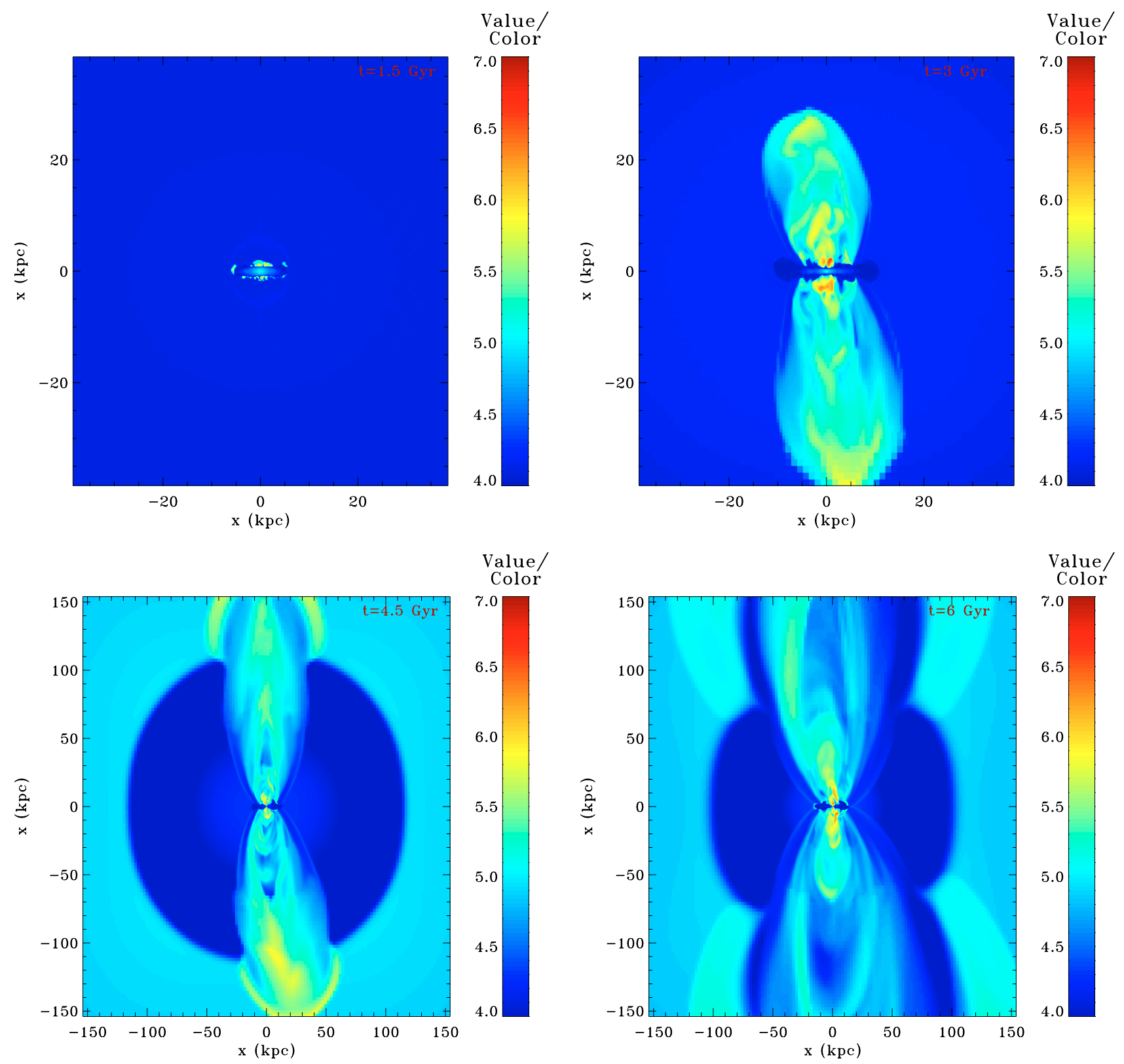

Fig. 2. Cut of the gas temperature in the $\mathrm{O} y z$ plane for the Sd simulation at different epochs. The two top panels are a 4 times zoom of the simulation box and the two bottom panels are the entire simulation box. The colour scale gives the temperature in log(K). Note that length scales are not the same in each panel.

determined by the accretion of gas infalling from the outer halo, as predicted by Eq. (7).

Figure 2 shows a map of the gas temperature in a cut perpendicular to the galactic plane for run $\operatorname{Sd}\left(10^{10} M_{\odot}\right.$ with $\lambda=0.1$ and $t_{0}=8 \mathrm{Gyr}$ ) at different epochs. The first panel, at $t=1.5 \mathrm{Gyr}$, shows no sign of wind, although it already corresponds to the peak of star formation in this galaxy (around $0.3 M_{\odot} / \mathrm{yr}$ ). We see several hot features trying to break out of the cold disc, but none are able to expand significantly, because of the ram pressure of infalling halo material. The second panel corresponds to a later epoch when the SFR has dropped by $50 \%$, down to $0.2 M_{\odot} / \mathrm{yr}$ (see also Fig. 3). Nevertheless, since gas accretion has also dropped significantly, two hot and large cavities now emerge out of the disc and propagate in the halo. At $t=4.5 \mathrm{Gyr}$ the galactic wind has finally escaped the halo outer boundaries, and is now in a quasi-permanent regime. These two large cavities have created some kind of tunnel, facilitating the ejection of hot, $\mathrm{SNe}$-driven material from the inner disc, all the way out to the IGM. At $t=6 \mathrm{Gyr}$, we see a final snapshot of the galactic wind, with a typical nozzle-like shape (see Figs. 2 and 3). The hot, metal-rich outflowing gas is surrounded by a dense shell of compressed and cooling halo gas. This dense shell will eventually fragment into small clouds which will fall back to the disc. We believe that this dense shell corresponds to the structure seen in many observations around M 82 (Heckman et al. 1990; Shopbell \& Bland-Hawthorn 1998; Martin 1998; Hoopes et al. 2003). This also corresponds to the "superwind" geometry described in Tenorio-Tagle \& Munoz-Tunon (1998) (see also Veilleux et al. 2005, and references therein). 

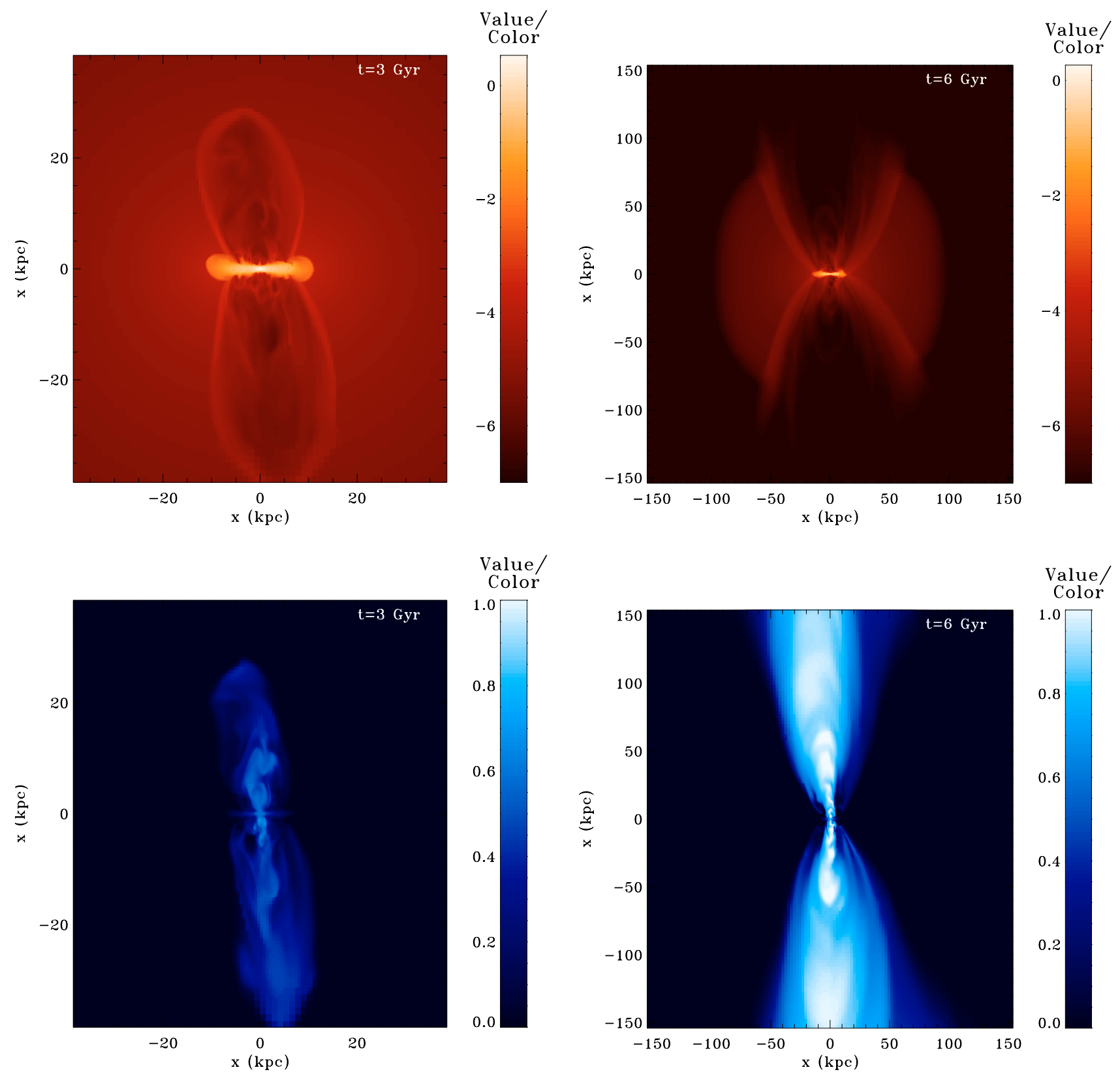

Fig. 3. Cut of the gas density (up) and metallicity (bottom) in the Oyz plane for the Sd simulation at different epochs. The two left panels are a 4 times zoom of the simulation box and the two right panels are the entire simulation box. The colour scale gives the density in $\log \left(\mathrm{cm}^{-3}\right)(u p)$ and the metallicity in $Z_{\odot}($ bottom $)$. Note that length scales are not the same in each panel.

\subsection{Wind formation epoch}

To describe the outflow more quantitatively, we define the net mass flux across a shell of radius $r_{\min }$ and thickness $\Delta r=r_{\max }-$ $r_{\min }$ as

$F=\int_{r_{\min }}^{r_{\max }} \frac{\rho \boldsymbol{u} \cdot \boldsymbol{n}}{\Delta r} 4 \pi r^{2} \mathrm{~d} r=F_{+}+F_{-}$,

where $\boldsymbol{n}=\boldsymbol{r} / r$. This net flux is further split into two different contributions: the positive flux, $F_{+}$, corresponding only to outflowing volume elements, namely those that satisfy $\boldsymbol{u} \cdot \boldsymbol{n}>0$, and the negative flux, $F_{-}$, corresponding to inflowing volume elements, satisfying $\boldsymbol{u} \cdot \boldsymbol{n}<0$. We focus here on the positive flux, in order to detect the outflow, and to estimate the amount of gas expelled as a function of time. The shell thickness was set to $\Delta r=2 r_{\mathrm{s}}$, and we vary the shell radius from $5 r_{\mathrm{s}}$ to $20 r_{\mathrm{s}}$, to define a proper radius where the outflow can be detected.
Figure 5 shows the positive flux measured at 4 different radii for our 4 low mass galaxies, as a function of time. The wind is detected when the positive flux rises sharply from zero to its maximum value. The smaller the shell radius is, the earlier the wind is detected. For our smallest shell radius, $r_{\text {min }}=5 r_{\mathrm{s}}$, the measured mass flux remains quasi constant in time, while for larger shell radii, the measured mass flux sharply rises to a much larger value, namely 2 to 3 times the inner flux, then slowly decays to the correct wind mass flux. This means that when the wind breaks out of the halo, a significant fraction (up to $\sim 2 / 3$ ) of the wind is filled by the hot gaseous halo and expelled out of the halo boundaries. When the permanent regime sets in later, the mass flux is roughly equal to the inner one, independent of the radius of the shell. We therefore consider only the case $r_{\min }=5 r_{\mathrm{s}}$ as the correct proxy for measuring the mass outflow rate in the wind. 


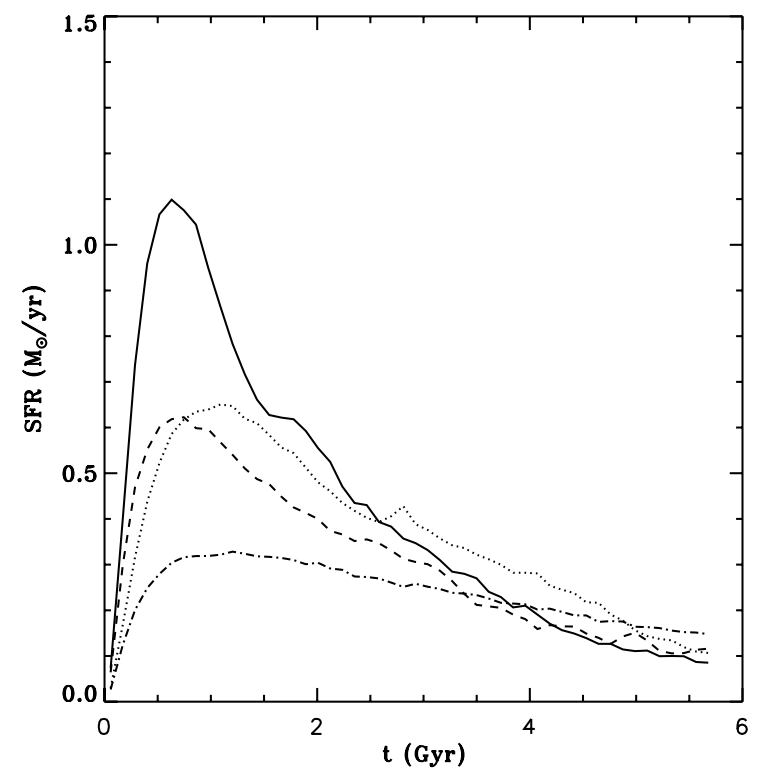

Fig. 4. SFRs of the $10^{10} M_{\odot}$ halos with $\lambda=0.04$ and $t_{0}=3$ Gyr (solid line), $\lambda=0.04$ and $t_{0}=8 \mathrm{Gyr}$ (dotted line), $\lambda=0.1$ and $t_{0}=3 \mathrm{Gyr}$ (dashed line) and $\lambda=0.1$ and $t_{0}=8 \mathrm{Gyr}$ (dash-dotted line).

We have plotted the positive flux at $r_{\min }=5 r_{\mathrm{s}}$ for our 4 galaxies in Fig. 6. The highest SFR galaxy, Sa, corresponds to our earliest wind formation epoch, around $t_{\mathrm{w}} \simeq 1 \mathrm{Gyr}$. According to our analytical model (see Fig. 1), this corresponds to the hydrodynamical efficiency parameter $\chi \simeq 0.9 \%$. Although runs $\mathrm{Sb}$ and $\mathrm{Sc}$ have identical star formation history (see Fig. 4), they have different wind formation epochs, respectively $t_{\mathrm{w}} \simeq 2$ and 3 Gyr. This demonstrates that for a given SFR, a more compact disc is more efficient in forming galactic winds. According to our analytical recipe, run $\mathrm{Sb}$ corresponds to $\chi \simeq 0.8 \%$, while run $\mathrm{Sc}$ barely reaches $\chi \simeq 0.6 \%$. The final galaxy, $\mathrm{Sd}$, features the latest wind to develop, with $t_{\mathrm{w}} \simeq 4 \mathrm{Gyr}$, corresponding also to a slightly lower hydrodynamical efficiency $\chi \simeq 0.7 \%$. The main conclusion of this section is that the conversion efficiency of the $\mathrm{SNe}$ energy deposition rate into wind luminosity is rather low in this model, around $\chi \simeq 1 \%$. This efficiency is directly related on the choice of the SNe energy injection rate, a quite uncertain parameter depending on the scale injection energy (here chosen equal to 50\%). We can see directly from Fig. 1 that, according to our numerical experiments, no wind will ever form in higher mass halo, as is demonstrated in the next section.

\subsection{Wind efficiency and metallicity}

Before describing the simulation results for the $10^{11} M_{\odot}$ halo, we would like to characterise the physical properties of the galactic winds we have obtained. It is common to define the wind efficiency as $\eta_{\mathrm{w}}=F_{+} / \dot{M}_{*}$. In other words, the mass outflow rate is expressed in units of the global SFR. From Figs. 4 and 5, we can compute directly $\eta_{\mathrm{w}}$ : we find the maximum efficiency at late time, when the permanent regime is settled, with values ranging from $\eta_{\mathrm{w}} \simeq 0.05$ for $\mathrm{Sc}$ and $\mathrm{Sd}$, up to $\eta_{\mathrm{w}} \simeq 0.1$ for $\mathrm{Sa}$ and $\mathrm{Sb}$. Here again, it appears clearly that more compact discs (low spin parameter $\lambda_{0}$ ) give more efficient winds, while extended discs (high spin parameter) are less efficient. All these values are extremely low compared to the wind efficiencies observed by Martin (1999) in Lyman break galaxies with values greater than 1 . These high-redshift galaxies are likely massive starbursts, for which our quiescent approach does not apply. On the other hand, we have determined that compact star formation sites result in earlier and stronger winds. Nuclear starbursts can be natural candidates to host very efficient winds with $\eta_{\mathrm{w}} \geq 1$, although these bright galaxies are probably rather rare objects in the early universe.

As seen from Fig. 3, the hot gas carried away in the wind is highly enriched with metals $\left(Z_{\mathrm{w}} \simeq Z_{\odot}\right)$. We define the average wind metallicity as the positive flux of metals divided by the positive flux of mass $Z_{\mathrm{w}}=(\rho Z u)_{+} / F_{+}$. Figure 7 shows the mean metallicity of the wind for our various low mass galaxies. We see no clear trend of the wind metallicity as a function of disc size or star formation efficiency. We can only observe that the metallicity of the wind is about 0.5 to $1 Z_{\odot}$. This rather high value confirms that the wind comes mainly from the gas within the galactic disc which is directly enriched by exploding SNe.

We now briefly discuss how these winds might explain the observed metallicity of the IGM. We know from our simulations that the typical mass outflow rate is around $\dot{M}_{\mathrm{w}} \simeq 0.01 M_{\odot} / \mathrm{yr}$ (see Fig. 6), with an average wind metallicity of $Z_{\mathrm{w}} \simeq 1 Z_{\odot}$ and a typical wind velocity around $u_{\mathrm{w}} \simeq 300 \mathrm{~km} \mathrm{~s}^{-1}$ (see Fig. 13). If we assume that the IGM is photo-ionised to a temperature around $10^{4}-10^{5} \mathrm{~K}$, we can compute the volume $V$ occupied by the wind when the bubble stalls by pressure equilibrium with the IGM as

$\dot{M}_{\mathrm{w}} u_{\mathrm{w}}^{2} t \simeq \frac{P_{\mathrm{IGM}} V}{\gamma-1}$

assuming the gas is adiabatic. After $6 \mathrm{Gyr}$, the radius $R \simeq V^{1 / 3}$ of the expanding wind lies around $1 \mathrm{Mpc}$. This corresponds to a swept-up mass of baryons roughly $5 \times 10^{9} M_{\odot}$. If we assume that the metals carried out by the wind perfectly mix with the zero-metallicity IGM, we obtain for the metallicity of the IGM the following very simple relation

$Z_{\mathrm{IGM}} \simeq\left(\frac{c_{\mathrm{s}}}{u_{\mathrm{w}}}\right)^{2} Z_{\odot} \simeq 10^{-3}-10^{-2} Z_{\odot}$

where $c_{\mathrm{s}} \simeq 10-30 \mathrm{~km} \mathrm{~s}^{-1}$ is the sound speed of the IGM. Note that this analysis is valid only for the idealised case we have considered here. Observations of the Ly $\alpha$ forest clouds show that they are metal enriched from $Z \simeq 10^{-3}$ up to $10^{-2} Z_{\odot}$ (Songaila \& Cowie 1996; Ellison et al. 2000). Cosmological simulations also confirm this trend, although the various wind models proposed (including ours) might be difficult to reconcile (Gnedin 1998; Thacker et al. 2002).

\section{4. $10^{11} M_{\odot}$ halo}

Figure 8 shows the star formation history for the 4 galaxies hosted by the $10^{11} M_{\odot}$ halo. They are qualitatively very similar to the $10^{10} M_{\odot}$ case, with La and Ld as the most extreme models, and $\mathrm{Lb}$ and $\mathrm{Lc}$ being quasi undistinguishable. Each galaxy has however a peak SFR which is $50 \%$ higher than its rescaled $10^{10} M_{\odot}$ counterpart. The circular velocity in these new galaxies is higher than the low mass ones, while the gas sound speed, because of the polytropic multiphase model, remains roughly constant, around $10 \mathrm{~km} \mathrm{~s}^{-1}$, so that the disc is now much thinner. This results in higher total star formation efficiencies. Using our analytical model, we can reproduce the 3 star formation histories using for run $\mathrm{Sa}, \mathrm{Sb}$ (and $\mathrm{Sc}$ ) and $\mathrm{Sd}$ the values $t_{*} \simeq 0.75,1.5$ and 3 Gyr respectively.

The other striking differences with low mass galaxies is the clear presence of an accretion shock surrounding the disc, and the notable absence of galactic winds. We observe instead a good 

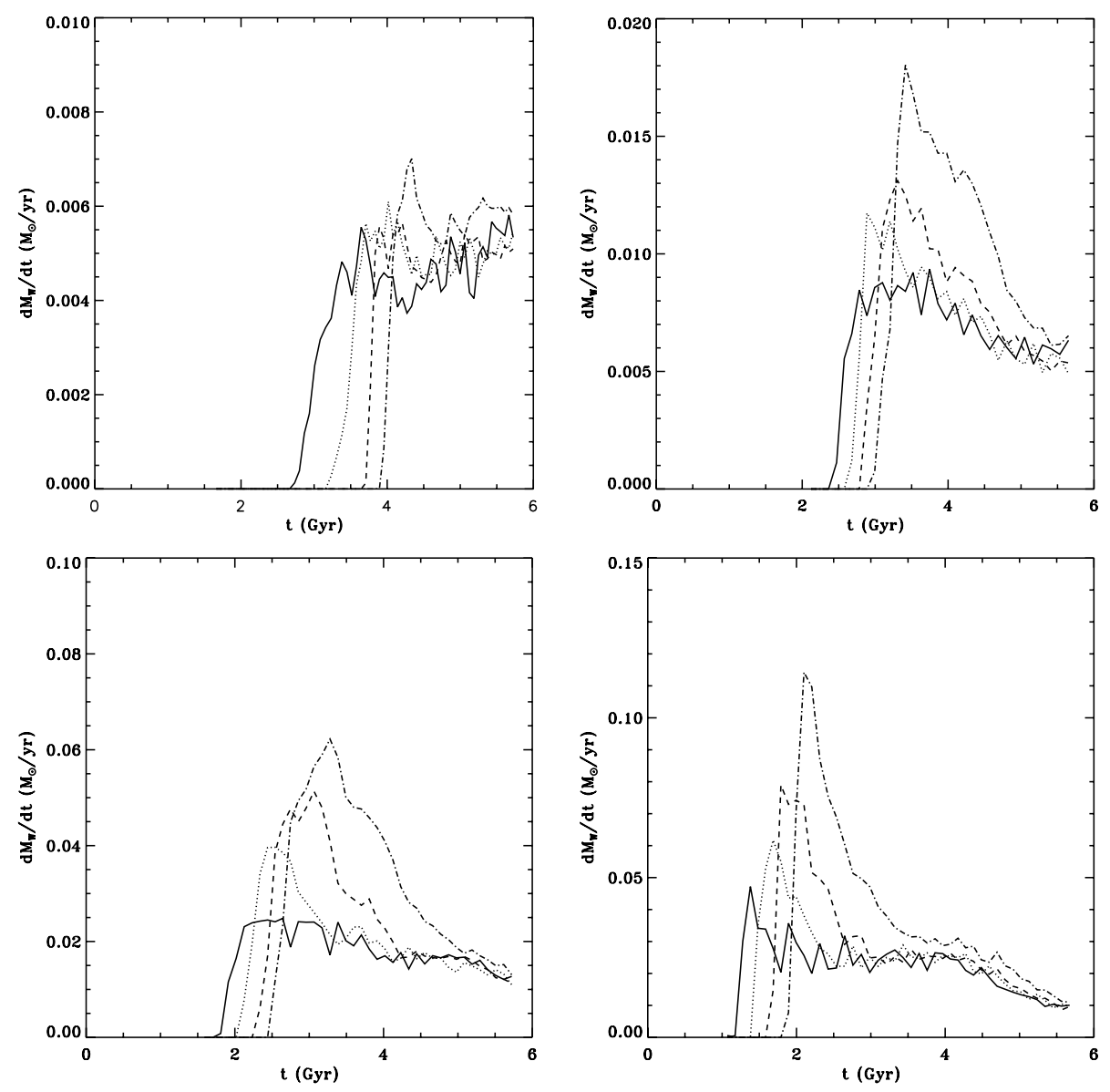

Fig. 5. Mean flux of mass outflowing calculated for the $10^{10} M_{\odot}$ halos with $\lambda=0.1$ and $t_{0}=8 \mathrm{Gyr}$ (top left panel), $\lambda=0.1$ and $t_{0}=3$ Gyr (top right panel), $\lambda=0.04$ and $t_{0}=8 \mathrm{Gyr}$ (bottom left panel) and $\lambda=0.04$ and $t_{0}=3 \mathrm{Gyr}$ (bottom right panel), and computed at different radius of the halo: $r=\left[5 r_{\mathrm{s}} ; 7 r_{\mathrm{s}}\right]$ (solid line), $r=\left[9 r_{\mathrm{s}} ; 11 r_{\mathrm{s}}\right]$ (dotted line), $r=\left[14 r_{\mathrm{s}} ; 16 r_{\mathrm{s}}\right]$ (dashed line), $r=\left[19 r_{\mathrm{s}} ; 21 r_{\mathrm{s}}\right]$ (dash-dotted line).

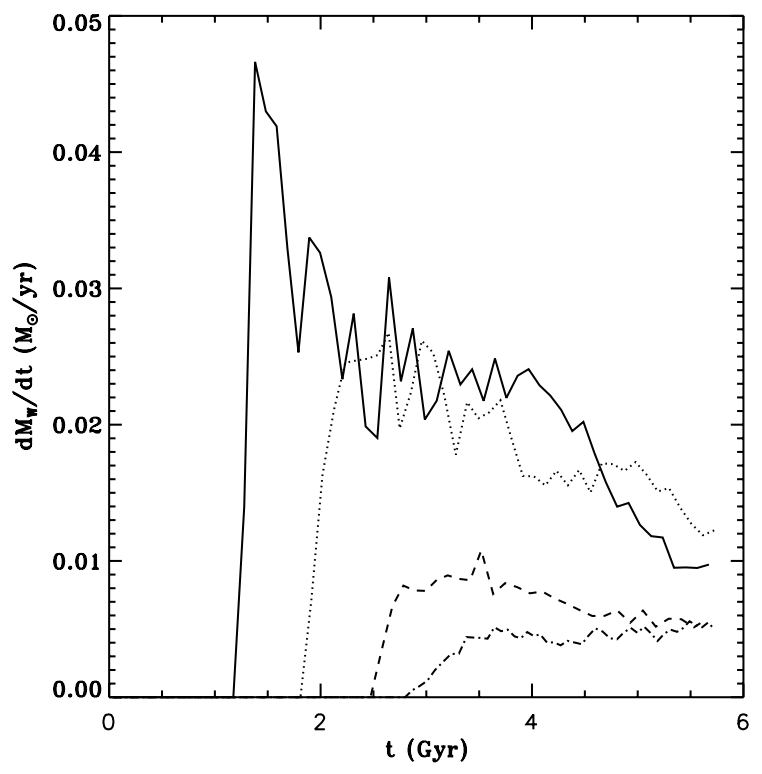

Fig. 6. Mean flux of mass outflowing calculated between $r=\left[5 r_{\mathrm{s}} ; 7 r_{\mathrm{s}}\right]$ for the $10^{10} M_{\odot}$ halos with $\lambda=0.04$ and $t_{0}=3 \mathrm{Gyr}$ (solid line), $\lambda=0.04$ and $t_{0}=8 \mathrm{Gyr}$ (dotted line), $\lambda=0.1$ and $t_{0}=3 \mathrm{Gyr}$ (dashed line) and $\lambda=0.1$ and $t_{0}=8 \mathrm{Gyr}$ (dash-dotted line).

galactic fountain, with plumes of hot gas rising above the disc, cooling down and falling back to the disc (see Fig. 9).

The formation of an accretion shock above a certain halo mass is now well understood: the stability analysis of a radiative shock wave, performed by Birnboim \& Dekel (2003), explains why for low mass halo no accretion shock forms. The mass

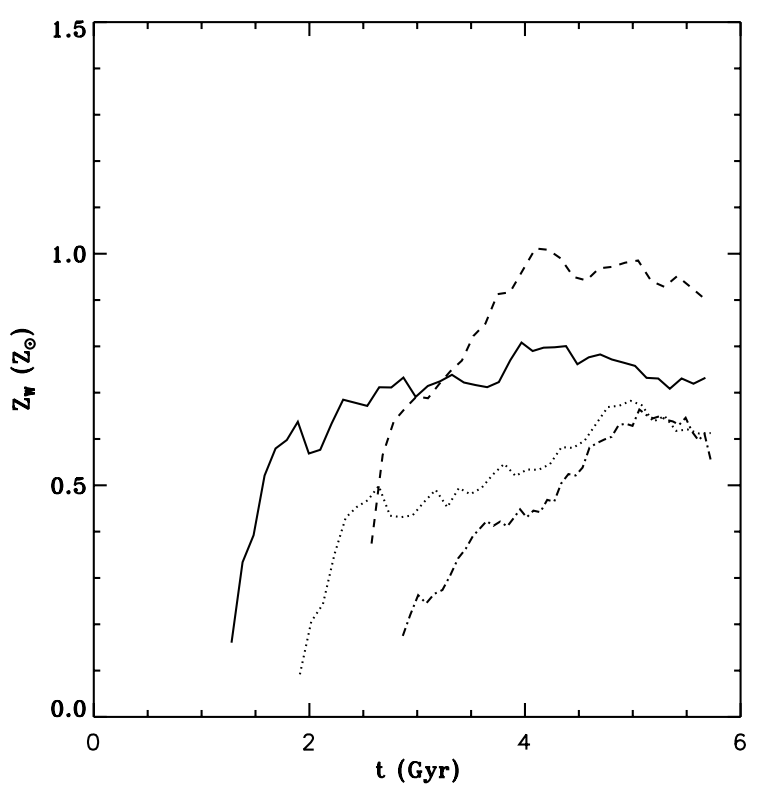

Fig. 7. Mean metallicity of the wind calculated between $r=\left[5 r_{\mathrm{s}} ; 7 r_{\mathrm{s}}\right]$ for the $10^{10} M_{\odot}$ halos with $\lambda=0.04$ and $t_{0}=3 \mathrm{Gyr}$ (solid line), $\lambda=0.04$ and $t_{0}=8 \mathrm{Gyr}$ (dotted line), $\lambda=0.1$ and $t_{0}=3 \mathrm{Gyr}$ (dashed line) and $\lambda=0.1$ and $t_{0}=8 \mathrm{Gyr}$ (dash-dotted line).

threshold above which the shock forms depends on the metallicity of the cooling gas. We see from Fig. 9 that in our simulation the shock heated gas above and below the disc is metal poor: in this case, Birnboim \& Dekel (2003) found a critical mass for shock appearance slightly below $10^{11} M_{\odot}$ for a zero metallicity 


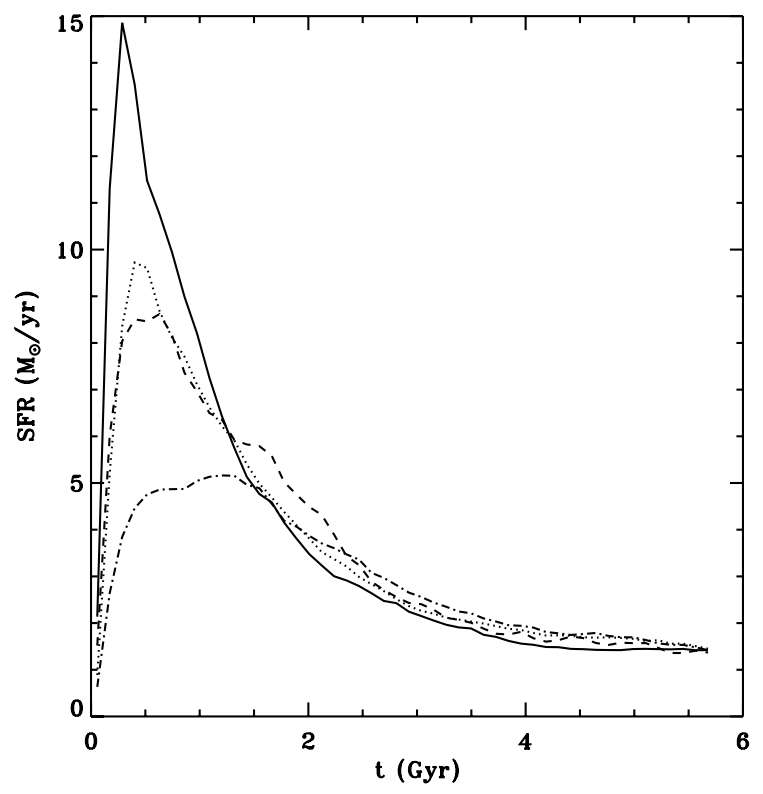

Fig. 8. SFRs of the $10^{11} M_{\odot}$ halos with $\lambda=0.04$ and $t_{0}=3 \mathrm{Gyr}$ (solid line), $\lambda=0.04$ and $t_{0}=8 \mathrm{Gyr}$ (dotted line), $\lambda=0.1$ and $t_{0}=3 \mathrm{Gyr}$ (dashed line) and $\lambda=0.1$ and $t_{0}=8 \mathrm{Gyr}$ (dash-dotted line).

gas, in good agreement with our numerical experiment. The accretion shock converts the infall kinetic energy into internal energy, so that our analytical treatment of wind formation remains valid, replacing the ram pressure by the thermal pressure. The accretion shocks reaches a maximum radius of $\sim 75 \mathrm{kpc}$ which is $\sim 2 / 3 r_{\text {vir }}$, almost the same virial radius fraction as that found in Birnboim \& Dekel (2003) for the cooling case after $4-5$ Gyr, and finally contracts down to $\sim 50 \mathrm{kpc}$ later. Although the disc is strongly perturbed by SNe blast waves, no galactic winds can form, in agreement with our simple analytical prediction, if we assume for the hydrodynamical efficiency $\chi \simeq 1 \%$, a value close to that obtained in the $10^{10} M_{\odot}$ case. The atmosphere above and below the disc is however highly turbulent and perturbed by buoyantly rising plumes. This convective flow is more visible in the metallicity map of Fig. 10.

Metals are confined within a rather small distance relative to the disc plane, namely $\sim 20 \mathrm{kpc}$. In this case, the thermal pressure of the shock-heated halo gas is very efficient in confining the galactic wind. Figure 11 compares the metallicity profiles of the gas between the $10^{10} M_{\odot}$ halo and the $10^{11} M_{\odot}$ halo (run $\mathrm{Sd}$ versus run $\mathrm{Ld}$ ) at different epochs. We define for that purpose a mass-weighted average metallicity as a function of height and a mass-weighted metallicity as a function of radius as

$Z(z)=\frac{\int_{0}^{r_{\mathrm{d}}} \rho Z 2 \pi r \mathrm{~d} r}{\int_{0}^{r_{\mathrm{d}}} \rho 2 \pi r \mathrm{~d} r}$ and $Z(r)=\frac{\int_{-h_{\mathrm{d}}}^{+h_{\mathrm{d}}} \rho Z 2 \pi \mathrm{d} z}{\int_{-h_{\mathrm{d}}}^{+h_{\mathrm{d}}} \rho 2 \pi \mathrm{d} z}$

where $\rho$ is the gas density and $Z$ is the gas metallicity. The disc radius $r_{\mathrm{d}}$ is taken equal to $r_{\mathrm{s}}$ and the disc scale height $h_{\mathrm{d}}=r_{\mathrm{d}} / 10$. In the wind case, metals are brought about at very large distance above and below the disc in the wind nozzle. The metallicity is roughly solar and tends to saturate at this value. In the fountain case, the metallicity in the halo remains rather low, roughly one tenth solar. On the other hand, the gas metallicity in the central part of the disc is significantly higher than in the wind case $\left(Z_{\text {gas }} \simeq 1.2 Z_{\odot}\right.$ versus $Z_{\text {gas }} \simeq 0.6 Z_{\odot}$, see Fig. 11$)$. This could be interpreted as the effect of the wind removing metals preferentially from the bulge of the galaxy. In the outer parts of the disc, however, the gas metallicity is very similar in both cases. This could also be interpreted as a higher star formation efficiency in the high mass case, especially in the central parts where the density is higher. As explained in great detail in Dalcanton (2006), it is quite difficult to disentangle the influence of star formation, gas infall and winds on metal enrichment in galaxies. We believe that, even in our idealised case, the metal content in our galaxies is determined by a subtle balance between these three processes.

\subsection{Stellar distribution and metallicity}

Figure 12 shows the surface density profile of stars and the corresponding mass-weighted average metallicity as a function of the disc radius. In both cases, we see a clear exponential disc, with a bulge-like feature in the central part and a sharp decline of surface density in the outer part. We find a bulge radius of roughly $1 \mathrm{kpc}$ and a disc exponential radius of $2.5 \mathrm{kpc}$ for the low mass system and of $4 \mathrm{kpc}$ for the high mass. We note that the outer radius of the low mass case $(8 \mathrm{kpc})$ in smaller than the rescaled outer radius of its high-mass counterpart $(10 \mathrm{kpc})$. This is due to the different disc thicknesses, so that for a given surface density, the threshold for star formation is reached more easily by the most massive galaxy. This confirms the fact that the polytropic multiphase model has a great importance in regulating the gaseous disc properties and therefore star formation efficiencies. The break in the stellar surface density corresponds roughly to the average radius where the gas reaches the density threshold of star formation $\left(\rho_{0}=0.1 \mathrm{H} \mathrm{cm}^{-3}\right)$. Note that the overall number of stars is very similar in both cases, demonstrating that the presence of the galactic wind has no impact on the amount of baryons locked into long-lived stars. This is consistent with the rather low wind efficiencies we have measured $\left(\eta_{\mathrm{w}} \simeq 10 \%\right)$. Our simulations will not solve the overcooling problem. More importantly, the stellar metallicity seems also to be rather insensitive to the "wind versus fountain" scenario. We have $Z_{*} \simeq 0.4 Z_{\odot}$ in the central part of our $10^{11} M_{\odot}$ halo galaxy, while we have $Z_{*} \simeq 0.3 Z_{\odot}$ in the low mass case. Note that we have computed a mass-weighted stellar metallicity so we are more sensitive to the overall star formation history rather than to the latest OB stars formed. Nevertheless, our wind model has no dramatic impact on the simulated stellar population. although there is a weak trend for low mass galaxies to have slightly smaller stellar metallicities than high mass. Here again, as in Dalcanton (2006), it is quite difficult to conclude whether this trend is due to a wind or to a smaller star formation efficiency.

\subsection{Gas kinematics}

The most striking and observable differences between the wind and the fountain can be found in the gas kinematics in the halo atmosphere, below and above the disc. In order to mimic as much as possible the observational signatures of our simulated galaxies, as seen by a spectral lines analysis from a distant observer whose line of sight is aligned with the disc rotation axis, we define the average metal velocity as

$v_{\perp}(z)=\frac{\int_{0}^{r_{\mathrm{d}}} \rho Z v_{z} 2 \pi r \mathrm{~d} r}{\int_{0}^{r_{\mathrm{d}}} \rho Z 2 \pi r \mathrm{~d} r} \quad$ and $v_{\perp}^{2}(z)=\frac{\int_{0}^{r_{\mathrm{d}}} \rho Z v_{z}^{2} 2 \pi r \mathrm{~d} r}{\int_{0}^{r_{\mathrm{d}}} \rho Z 2 \pi r \mathrm{~d} r}$.

The velocity dispersion and the sound speed are defined as usual by

$\sigma_{\perp}^{2}(z)=v_{\perp}^{2}(z)-v_{\perp}(z)^{2}$ and $c_{\mathrm{s}}^{2}(z)=\frac{\int_{0}^{r_{\mathrm{d}}} \rho Z c_{\mathrm{s}}^{2} 2 \pi r \mathrm{~d} r}{\int_{0}^{r_{\mathrm{d}}} \rho Z 2 \pi r \mathrm{~d} r}$ 

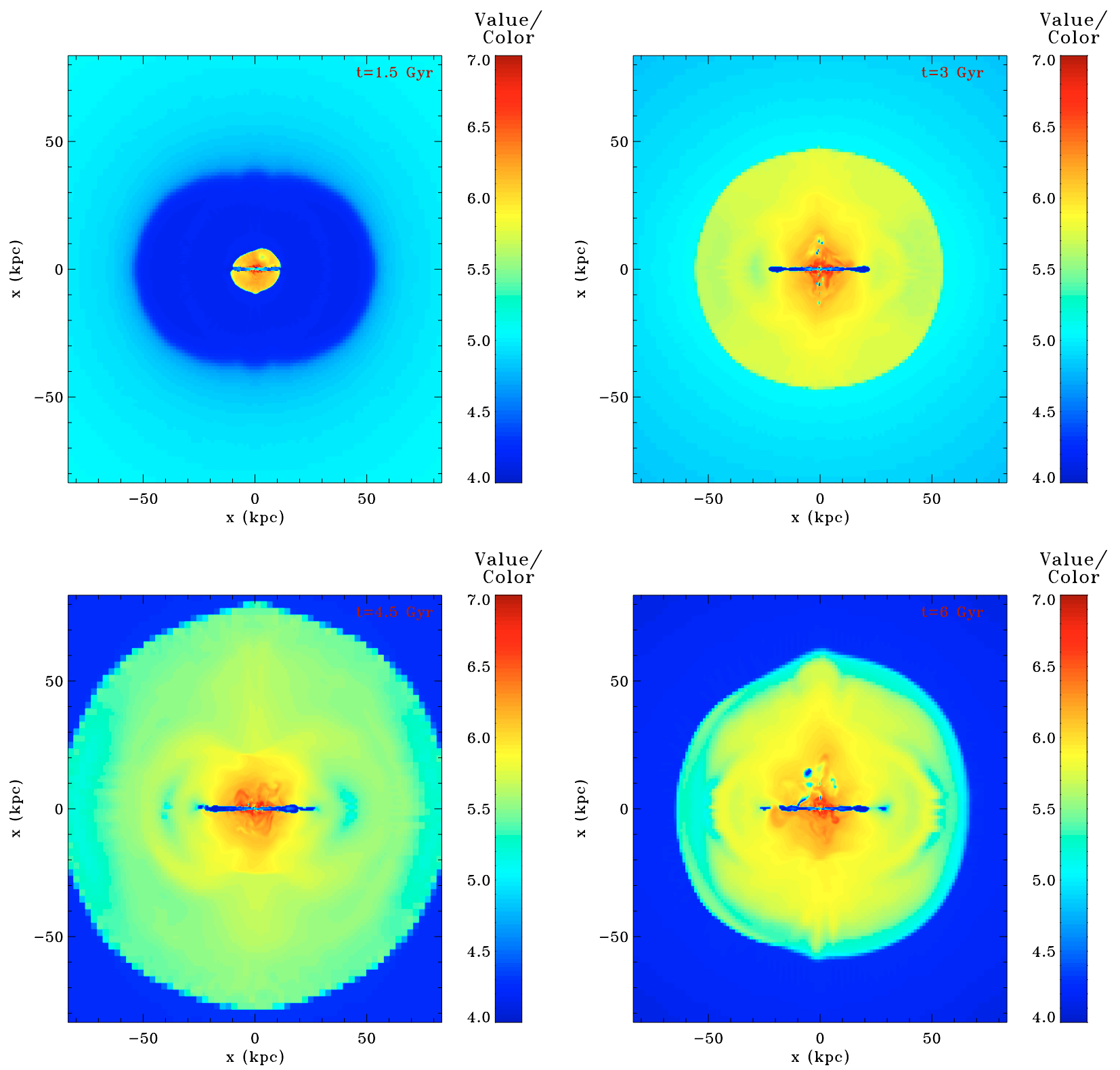

Fig. 9. Cut of the gas temperature in the Oyz plane for the Ld simulation at different epochs. The 4 panels are a 4 times zoom of the simulation box. The colour scale gives the temperature in $\log (\mathrm{K})$. Note that length scales are not the same in each panel.

Figure 13 shows these various profiles as a function of height for the wind case (left) and the fountain case (right). In the former case, we clearly see a strong symmetrical expansion of the metals, with a strong acceleration up to $200 \mathrm{~km} \mathrm{~s}^{-1}$ in $10 \mathrm{kpc}$, then a plateau afterwards when the wind reaches its terminal velocity. In the latter case, on the contrary, we see a converging flow, with a velocity of the order of $100 \mathrm{~km} \mathrm{~s}^{-1}$, similar to the velocity of infalling halo material. At an altitude of $20 \mathrm{kpc}$, this bulk infall velocity declines and at the same time, the velocity dispersion rises: we are entering the galactic fountain for which the flow is subsonic and highly turbulent. Note that in the wind case, the velocity dispersion also rises up to $100 \mathrm{~km} \mathrm{~s}^{-1}$ as we approach the disc. This cannot be attributed to a turbulent flow, since it would have been supersonic (the sound speed is around $50 \mathrm{~km} \mathrm{~s}^{-1}$ ). It is in fact due to a geometric effect: as long as the wind expansion velocity remains subsonic, below an altitude of $10 \mathrm{kpc}$, the section of the wind increases with height. When the wind turns supersonic, around $z \simeq 10 \mathrm{kpc}$, the section of the wind is forced to remain constant. This process is responsible for the typical nozzle-like shape of the wind. At low altitude, since the wind is expanding quasi isotropically, this mimics a supersonic velocity dispersion which vanishes at higher altitude when the wind flow is quasi parallel to the galaxy rotation axis.

The $10^{10} M_{\odot}$ halo is characterised by a fast supersonic flow, expanding quasi-isotropically at low altitude but quasi-parallel to the rotation axis at higher altitude. The $10^{11} M_{\odot}$ halo, on the contrary, is characterised by a converging pattern quickly thermalised close to the disc in a subsonic turbulent flow.

We applied the method used by Prochaska et al. (2007) to test whether our simulations show velocity dispersion in the metal absorption lines of QSOs wider than the velocity dispersion expected from pure gravitational processes (of the order of the galaxy circular velocity). In this way, we can test whether our winds would have been detected. In their paper, Prochaska et al. (2007) use $\Delta v_{90}$ as the velocity interval encompassing $90 \%$ of the mass of a given metal absorber. We simulate an observer watching the galaxy along the direction of the wind and found that $90 \%$ of the mass of metals corresponds to metals with 

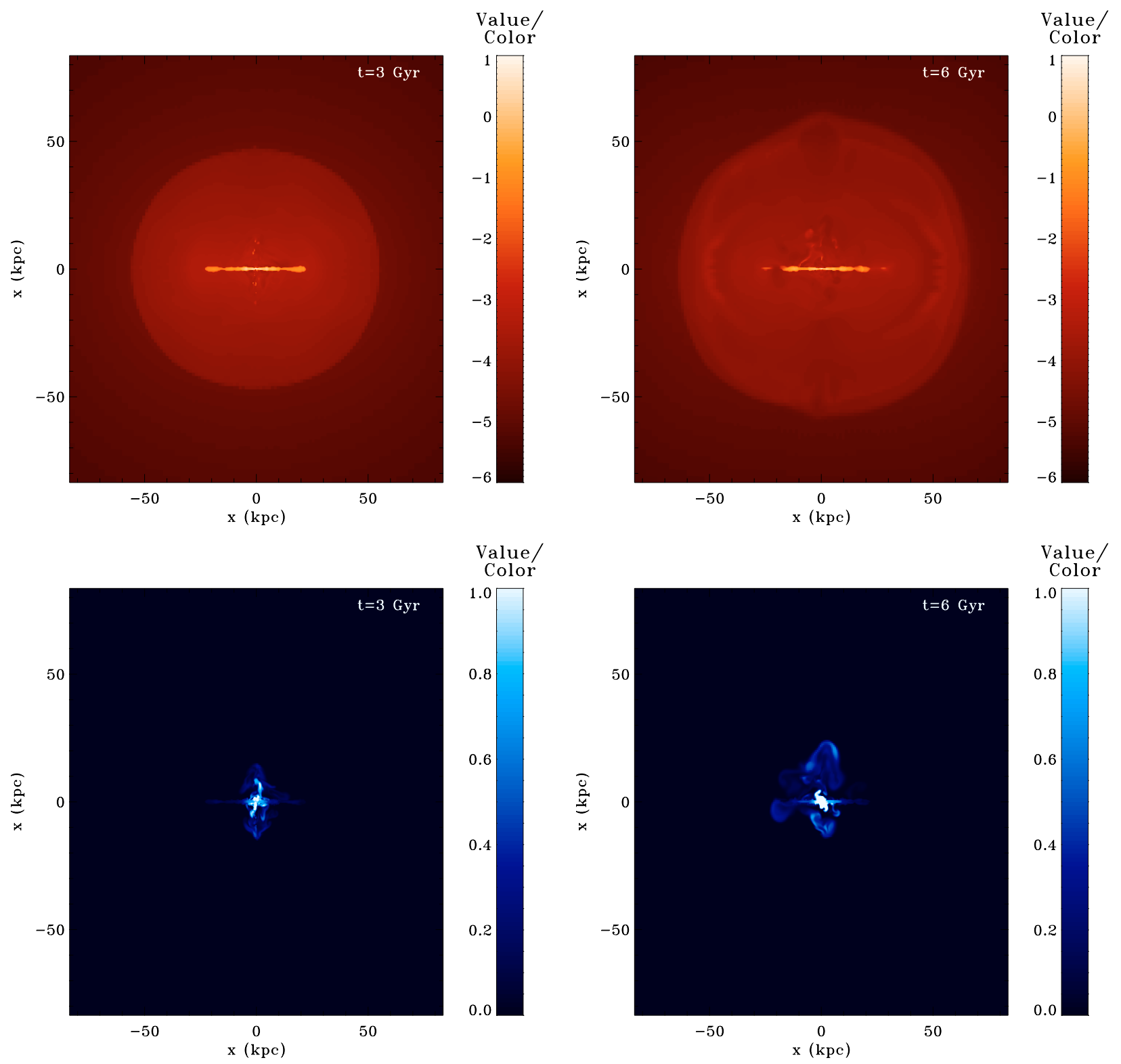

Fig. 10. Cut of the gas density (up) and metallicity (bottom) in the Oyz plane for the Ld simulation at different epochs. The two left panels are a 4 times zoom of the simulation box and the two right panels are the entire simulation box. The colour scale gives the density in $\log \left(\mathrm{cm}^{-3}\right)(u p)$ and the metallicity in $Z_{\odot}($ bottom). Note that length scales are not the same in each panel.

velocity lower than $5 \mathrm{~km} \mathrm{~s}^{-1}$. This is in agreement with our findings that wind efficiencies are very low in our model.

\section{Conclusion}

In comparison with Springel \& Hernquist (2003), we have very similar initial conditions but a lower wind for $\eta_{\mathrm{w}}$. We have therefore more difficulties solving the "over-cooling" problem. The explanation has two reasons: on the one hand Springel \& Hernquist (2003) model SNe explosions with a phenomenological approach so that every single ejecta in the disc entirely participates in the large scale outflow. In this work, our goal was to model SNe explosions with a self-consistent Sedov blast approach where ejectas are tightly coupled to the galactic disc (injecting energy in the galactic turbulent cascade). On the other hand, we have a cooling function depending on metallicity: this increases radiative losses significantly compared to Springel \& Hernquist (2003). Kobayashi et al. (2006) performed SPH simulations of isolated discs with star formation and selfconsistent $\mathrm{SNe}$ feedback, metal dependent cooling and the same set of initial conditions as ours. They observed very high mass ejection rates for the $10^{10} M_{\odot}$ halo with a total mass ejected rising to $80 \%$ of the total baryons mass. They noted however a strong dependance of their results on numerical resolution and, more importantly, on the size of the feedback radius (it can be as high as $10 \mathrm{kpc}$ !). They even observed a faint wind in the $10^{12} M_{\odot}$ halo case. Tasker \& Bryan (2006) have simulated a pre-formed isolated disc with the AMR code ENZO with star formation and self-consistent $\mathrm{SNe}$ feedback released in pure thermal form over one dynamical timescale. They succeed in shutting down star formation but they did not take into account infalling gas from the halo. Fujita et al. (2004) constructed an analytical prescription to constrain the mass of the halos that can form large-scale outflow with starburst physics. They have performed several sets of 2D grid simulations of a pre-formed galaxy with an imposed analytical infall model and found results very similar to ours (see 

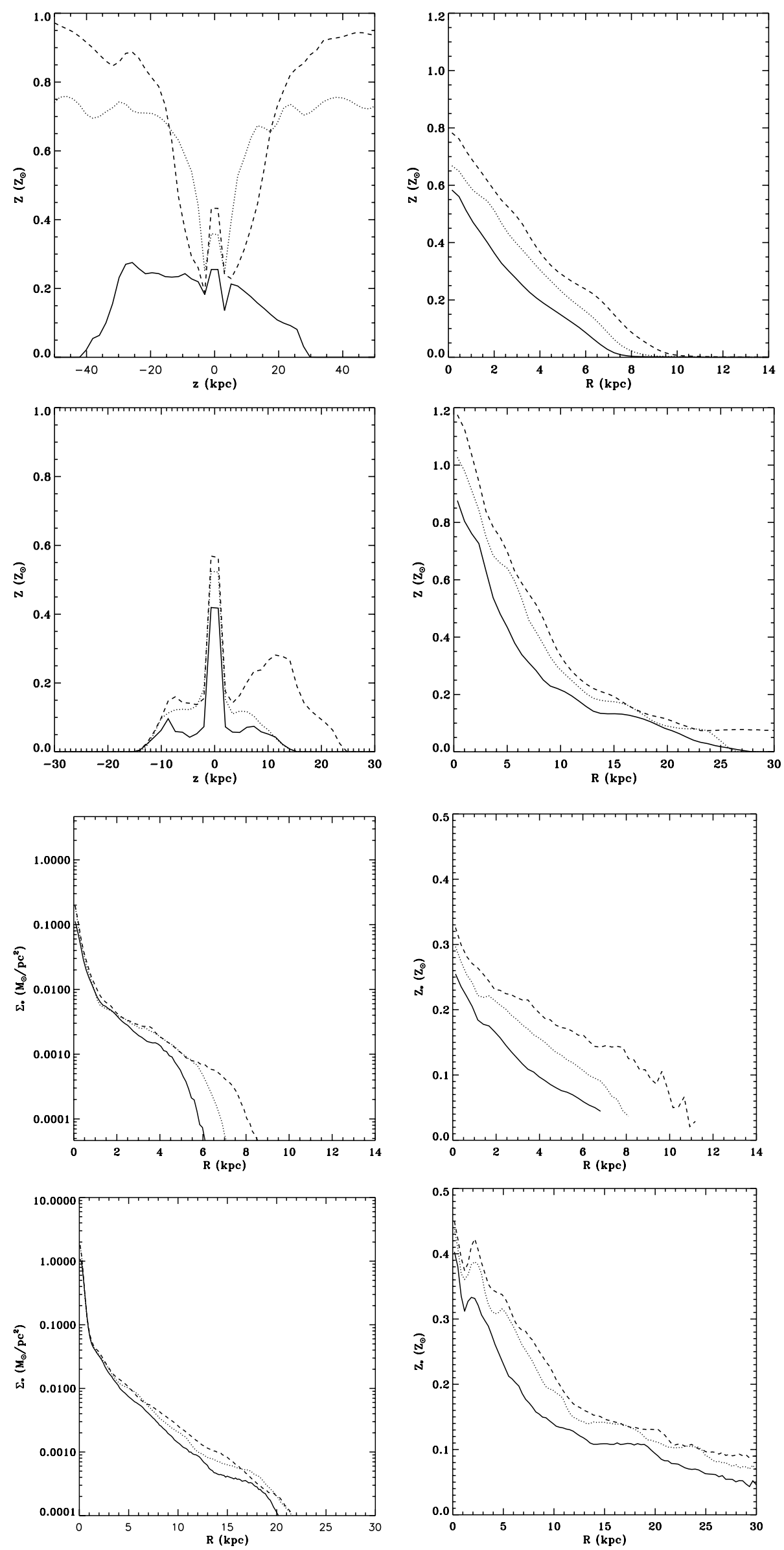

Fig. 11. Mean metallicity of the gas as a function of the projected height (left) and radius (right) for Sd simulation ( $u p$ ) and the Ld simulation (bottom) at different times: $t=3 \mathrm{Gyr}$ (solid line), $t=4.5 \mathrm{Gyr}$ (dotted line), $t=6 \mathrm{Gyr}$ (dashed line).

Fig. 12. Surface density (left) and mean metallicity (right) of the stars as a function of the projected radius for the Sd simulation (up) and the Ld simulation (bottom) at different times: $t=3 \mathrm{Gyr}$ (solid line), $t=4.5 \mathrm{Gyr}$ (dotted line), $t=6 \mathrm{Gyr}$ (dashed line). 

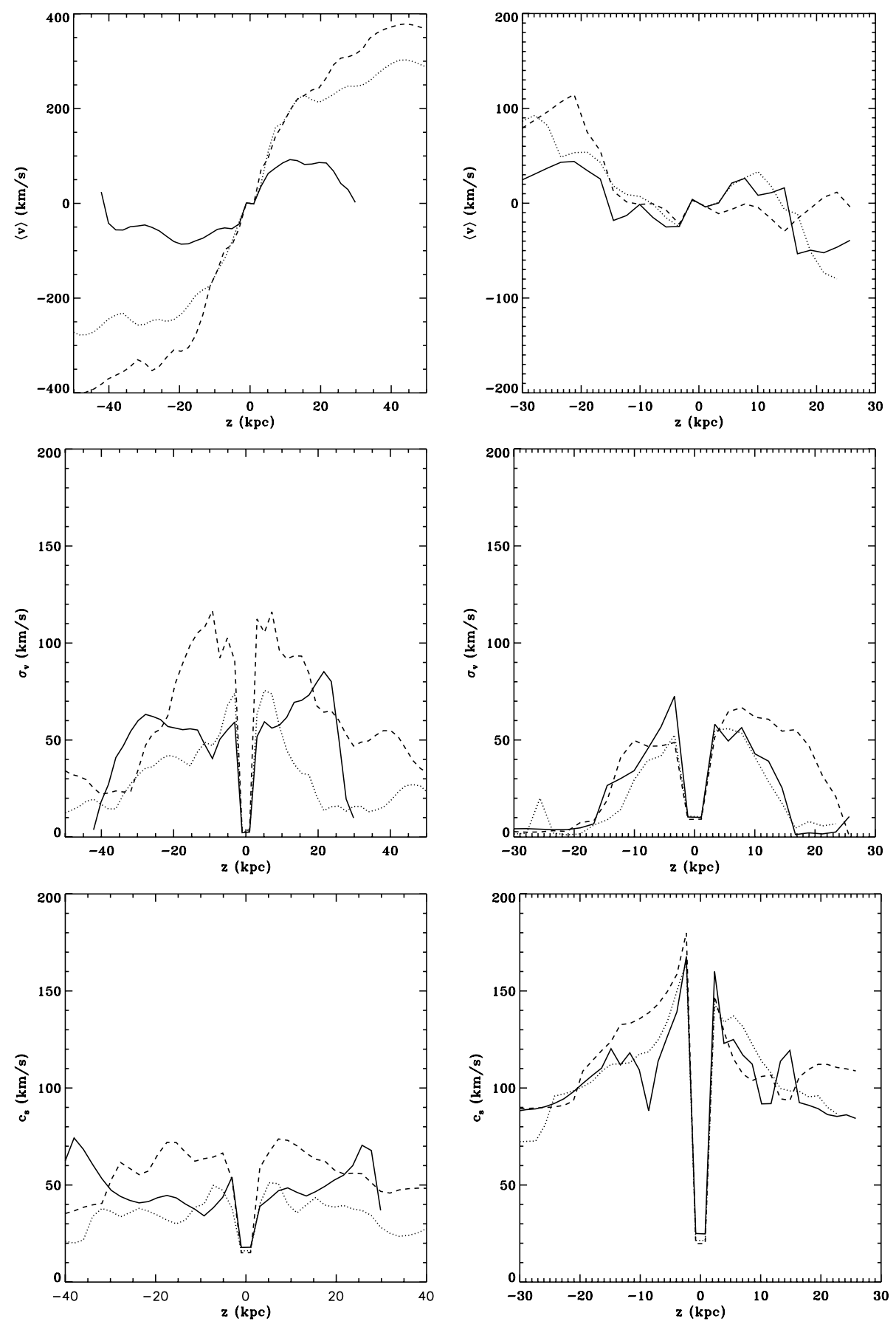

Fig. 13. Mean velocity (up), velocity dispersion (middle), sound speed (bottom) of the gas as a function of the projected height for the Sd simulation (left) and the Ld simulation (right) at different times: $t=3 \mathrm{Gyr}$ (solid line), $t=4.5 \mathrm{Gyr}$ (dotted line), $t=$ 6 Gyr (dashed line).

Fig. 1 in Fujita et al. 2004): the key role played by infall and a very low hydrodynamical efficiency for quiescent modes of star formation.

Using a quiescent model of star formation in isolated galaxies, self-consistently simulated from a cooling NFW halo, we have studied the conditions for a galactic wind to break out of the ram-pressure exerted by the infalling gas. Our simulations have shown that a galactic super wind forms in halos of $10^{10} M_{\odot}$ and no wind can form in halo of mass greater than $10^{11} M_{\odot}$, even for our most favourable couple of halo parameters $(\lambda=0.04$, $t_{0}=3 \mathrm{Gyr}$ ). For large galaxies, a galactic fountain appears and expels metal rich gas that cannot escape from the galaxy. Those results are consistent with our toy model which predicts no wind in those galaxies if the hydrodynamical efficiency is of a few percent as suggested by our simulations. Using this rather simple toy model, we understand this failure as being due to the ram pressure of infalling material confining the outflowing wind.

Although galactic winds develop in $10^{10} M_{\odot}$ halos, they are not sufficient to explain the "overcooling" problem. The mass ejection efficiency obtained in our simulations $\sim 3-12 \%$ is one order of magnitude lower than that observed by Martin (1999) in starburst galaxies. The main conclusion of this paper is that with a self-consistent treatment of $\mathrm{SNe}$ feedback in idealised simulations of quiescent star forming galaxies we cannot reproduce the observed mass ejection rates and therefore reduce the cold baryon fraction. On the contrary, SNe feedback is an efficient process for metal enrichment of the IGM. Cosmological simulations with $\mathrm{SNe}$ feedback could test this scenario in a more 
quantitative way. Using a more realistic cosmological setting may result into non-spherical accretion flows, and therefore a less stringent criterion for a wind to break out.

Considering that small mass galaxies preferentially form along filaments, non-spherical accretion should allow the wind to break out more easily and probably to be more efficient. For more massive galaxies, however, the accretion of gas into the disc is likely to proceed through a Virial shock, and not along filaments (Dekel \& Birnboim 2006). Our conclusions should therefore remain valid, so that massive galaxies should not provide winds powerful enough to enrich the IGM by SNe feedback (but other ways of enrichment such as gas stripping from satellites can be considered as in Schindler et al. 2005). Dekel \& Birnboim (2006) have computed the critical mass between filamentary accretion and Virial shocks in the range $10^{11}-10^{12} M_{\odot}$ depending on the metallicity of the infalling gas. Combining their results with ours, we can conclude that halos larger than a few $10^{11} M_{\odot}$ cannot produce galactic winds, even within a realistic cosmological environment. At higher redshift, accretion rates are believed to be higher than that we have considered here, so that galactic winds at earlier epochs are even more unlikely. A proper modelling of starburst (yet to be invented) might also provide an easier route for increasing the feedback efficiency of SNe-driven outflows.

Acknowledgements. This work has been supported by the Horizon Project. Computations were done at CCRT, the CEA Supercomputing Centre.

\section{Appendix A: Numerical scheme for supernovae}

We define the mass vanished in the star formation process as:

$\left(\Delta m_{\mathrm{g}}\right)_{\mathrm{SF}}=m_{*}\left(1+\eta_{\mathrm{SN}}+\eta_{\mathrm{W}}\right)$,

where the second term of the right part is equal to the total debris mass $m_{\mathrm{d}}$. We also introduced the mass loading factor $\eta_{\mathrm{W}}$, the parameter which determines the gas mass carried in debris. And $m_{*}$ is equal to

$m_{*}=\frac{\rho_{0} \delta x^{3}}{1+\eta_{\mathrm{SN}}+\eta_{\mathrm{W}}}$,

$\delta x^{3}$ is the volume of the cell where the star particle is spawned. Then if we introduce the fraction of debris particles:

$f_{\mathrm{d}}=\frac{\eta_{\mathrm{SN}}+\eta_{\mathrm{W}}}{1+\eta_{\mathrm{SN}}+\eta_{\mathrm{W}}}$

we can simplify Eq. (A.1) by saying that a fraction $f_{\mathrm{d}}$ of the gas consumed goes into debris particles, and a fraction $1-f_{\text {d }}$ goes into star particle.

The maximum speed of a debris is given by

$u_{\mathrm{d}}=\frac{u_{\mathrm{SN}}}{\sqrt{1+\eta_{\mathrm{W}} / \eta_{\mathrm{SN}}}}$.

$u_{\mathrm{SN}}$ is the typical velocity corresponding to the kinetic energy released in one single $\mathrm{SN}$ explosion $\left(u_{\mathrm{SN}} \simeq 3200 \mathrm{~km} \mathrm{~s}^{-1}\right)$.

Thus, the energy released to the gas by the debris is

$E_{\mathrm{d}}=\eta_{\mathrm{SN}} \frac{m_{*}}{M_{\mathrm{SN}}} E_{\mathrm{SN}}$.

$M_{\mathrm{SN}}$ and $E_{\mathrm{SN}}$ are respectively the typical progenitor mass and energy of an exploding type II SN (i.e. $M_{\mathrm{SN}}=10 M_{\odot}$ and $\left.E_{\mathrm{SN}}=10^{51} \mathrm{erg}\right)$. The energy is independent from the mass loading factor. Then, we have to compute the moment conservation for constraining this free parameter. We want to reproduce a Sedov blast wave, and so the velocity of the gas when the debris are coupled with it is the Sedov speed of the shock propagation.

$u_{\text {Sedov }}=\beta \frac{2}{5}\left(\frac{E_{\mathrm{d}}}{\rho_{0} \Delta x^{3}}\right)^{1 / 2}$,

$\beta$ is a multiplication factor nearly equal to 1 , we admit it is equal to $1, \rho_{0}$ is the density of the gas where the explosion takes place and $\Delta x$ is the radius of the shock from the centre of the explosion. We can also express $u_{\text {Sedov }}$ in terms of $u_{\mathrm{SN}}$ :

$u_{\mathrm{Sedov}}=\frac{\sqrt{2}}{5}\left[\eta_{\mathrm{SN}}\left(\frac{\delta x}{\Delta x}\right)^{3} \frac{1}{1+\eta_{\mathrm{SN}}+\eta_{\mathrm{W}}}\right]^{1 / 2} u_{\mathrm{SN}}$.

If the Sedov blast wave is only valid in homogeneous fluid, then it seems surprising to apply it in regions of star formation where the gas condenses in high overdensity regions of molecular clouds. This is the reality, but our simulations do not reproduce such high resolution features, and the assessment on the polytropic trend of the gas at high densities implies more pressurised regions in the disc. This behaviour at high densities transforms high over-density regions of star formation into smoother regions at the scale of one cell of the numerical grid. This is the reason that allows us to use the Sedov blast wave model. We want the gas to have the moment of a Sedov blast wave, so the conservation of the moment gives us:

$m_{\mathrm{d}} u_{\mathrm{d}}=\left(m_{\mathrm{g}}+m_{\mathrm{d}}\right) \bar{u}_{\text {Sedov }}$

where $m_{\mathrm{g}}=4 / 3 \pi \rho_{0} \Delta x^{3}$ is the gas mass at which the SN explosion occurs, and $\bar{u}_{\text {Sedov }} \simeq u_{\text {Sedov }} / 3$ is the mean velocity of the gas carried along the shock.

As we know all the quantities of this last equation, we can constraint our free parameter $f_{\mathrm{d}}$, and obtain

$\left[1+\frac{1}{f_{\mathrm{d}}} \frac{4}{3} \pi\left(\frac{\Delta x}{\delta x}\right)^{3}\right] \frac{\sqrt{2}}{15}\left[f_{\mathrm{d}}\left(\frac{\delta x}{\Delta x}\right)^{3}\right]^{1 / 2}=1$.

If we want our debris to propagate to the nearest cells, we assume that $\Delta x \simeq 1.5$, and find the optimal value for $f_{\mathrm{d}} \simeq 0.5$. Thus for a typical value of $\eta_{\mathrm{SN}}=0.1$ we find for the mass loading factor, our only free parameter, $\eta_{\mathrm{W}} \simeq 1$.

\section{References}

Abadi, M. G., Navarro, J. F., Steinmetz, M., \& Eke, V. R. 2003, ApJ, 591, 499 Adelberger, K. L., Steidel, C. C., Shapley, A. E., \& Pettini, M. 2003, ApJ, 584, 45

Ascasibar, Y., Yepes, G., Gottlöber, S., \& Müller, V. 2002, A\&A, 387, 396

Ascasibar, Y., Yepes, G., Müller, V., \& Gottlöber, S. 2003, MNRAS, 346, 731

Bertone, S., Stoehr, F., \& White, S. D. M. 2005, MNRAS, 359, 1201

Birnboim, Y., \& Dekel, A. 2003, MNRAS, 345, 349

Blanchard, A., Valls-Gabaud, D., \& Mamon, G. A. 1992, A\&A, 264, 365

Bland, J., \& Tully, B. 1988, Nature, 334, 43

Bouché, N., Lehnert, M. D., \& Péroux, C. 2006, MNRAS, 367, L16

Bullock, J. S., Dekel, A., Kolatt, T. S., et al. 2001, ApJ, 555, 240

Cen, R., \& Bryan, G. L. 2001, ApJ, 546, L81

Cen, R., \& Ostriker, J. P. 1992, ApJ, 399, L113

Cioffi, D. F., McKee, C. F., \& Bertschinger, E. 1988, ApJ, 334, 252

Cole, S., Lacey, C. G., Baugh, C. M., \& Frenk, C. S. 2000, MNRAS, 319, 168

Dalcanton, J. J. 2006, ArXiv Astrophysics e-prints

Dekel, A., \& Silk, J. 1986, ApJ, 303, 39

Dekel, A., \& Birnboim, Y. 2006, MNRAS, 368, 2

Ellison, S. L., Songaila, A., Schaye, J., \& Pettini, M. 2000, AJ, 120, 1175

Fujita, A., Mac Low, M.-M., Ferrara, A., \& Meiksin, A. 2004, ApJ, 613, 159

Gnedin, N. Y. 1998, MNRAS, 294, 407

Governato, F., Willman, B., Mayer, L., et al. 2006, ArXiv Astrophysics e-prints

Hatton, S., Devriendt, J. E. G., Ninin, S., et al. 2003, MNRAS, 343, 75 
Heckman, T. M., Armus, L., \& Miley, G. K. 1990, ApJS, 74, 833

Heckman, T. M., Lehnert, M. D., Strickland, D. K., \& Armus, L. 2000, ApJS, 129,493

Hoopes, C. G., Heckman, T. M., Strickland, D. K., \& Howk, J. C. 2003, ApJ, 596, L175

Hultman, J., \& Pharasyn, A. 1999, A\&A, 347, 769

Joung, M. K. R., \& Mac Low, M.-M. 2006, ApJ, 653, 1266

Katz, N. 1992, ApJ, 391, 502

Katz, N., Weinberg, D. H., \& Hernquist, L. 1996, ApJS, 105, 19

Kauffmann, G., Colberg, J. M., Diaferio, A., \& White, S. D. M. 1999, MNRAS, 303, 188

Kay, S. T., Pearce, F. R., Frenk, C. S., \& Jenkins, A. 2002, MNRAS, 330, 113

Kennicutt, Jr., R. C. 1998, ApJ, 498, 541

Kobayashi, C., Springel, V., \& White, S. D. M. 2006, ArXiv Astrophysics e-prints

Krumholz, M. R., \& Tan, J. C. 2007, ApJ, 654, 304

Larson, R. B. 1974, MNRAS, 169, 229

Mac Low, M.-M., \& Ferrara, A. 1999, ApJ, 513, 142

Maller, A. H., \& Dekel, A. 2002, MNRAS, 335, 487

Marri, S., \& White, S. D. M. 2003, MNRAS, 345, 561

Martin, C. L. 1998, ApJ, 506, 222

Martin, C. L. 1999, ApJ, 513, 156

McKee, C. F., \& Ostriker, J. P. 1977, ApJ, 218, 148

Melioli, C., \& de Gouveia Dal Pino, E. M. 2004, A\&A, 424, 817

Mihos, J. C., \& Hernquist, L. 1994, ApJ, 437, 611

Monaco, P. 2004, MNRAS, 352, 181

Mori, M., Yoshii, Y., Tsujimoto, T., \& Nomoto, K. 1997, ApJ, 478, L21

Navarro, J. F., \& Benz, W. 1991, ApJ, 380, 320

Navarro, J. F., \& White, S. D. M. 1993, MNRAS, 265, 271

Navarro, J. F., Frenk, C. S., \& White, S. D. M. 1996, ApJ, 462, 563

Okamoto, T., Eke, V. R., Frenk, C. S., \& Jenkins, A. 2005, MNRAS, 363, 1299

Pettini, M., Shapley, A. E., Steidel, C. C., et al. 2001, ApJ, 554, 981

Pettini, M., Rix, S. A., Steidel, C. C., et al. 2002, ApJ, 569, 742
Prochaska, J. X., Chen, H.-W., Wolfe, A. M., Dessauges-Zavadsky, M., \& Bloom, J. S. 2007, ArXiv Astrophysics e-prints

Rasera, Y., \& Teyssier, R. 2006, A\&A, 445,

Read, J. I., \& Gilmore, G. 2005, MNRAS, 356, 107

Robertson, B., Yoshida, N., Springel, V., \& Hernquist, L. 2004, ApJ, 606, 32

Salpeter, E. E. 1955, ApJ, 121, 161

Scannapieco, E., Thacker, R. J., \& Davis, M. 2001, ApJ, 557, 605

Scannapieco, C., Tissera, P. B., White, S. D. M., \& Springel, V. 2006, MNRAS, 371,1125

Schindler, S., Kapferer, W., Domainko, W., et al. 2005, A\&A, 435, L25

Shopbell, P. L., \& Bland-Hawthorn, J. 1998, ApJ, 493, 129

Shull, J. M., \& Silk, J. 1979, ApJ, 234, 427

Somerville, R. S., \& Primack, J. R. 1999, MNRAS, 310, 1087

Sommer-Larsen, J., Gelato, S., \& Vedel, H. 1999, ApJ, 519, 501

Sommer-Larsen, J., Götz, M., \& Portinari, L. 2003, ApJ, 596, 47

Songaila, A., \& Cowie, L. L. 1996, AJ, 112, 335

Springel, V., \& Hernquist, L. 2003, MNRAS, 339, 289

Steinmetz, M., \& Navarro, J. F. 1999, ApJ, 513, 555

Stinson, G., Seth, A., Katz, N., et al. 2006, ArXiv Astrophysics e-prints

Sutherland, R. S., \& Dopita, M. A. 1993, ApJS, 88, 253

Tasker, E. J., \& Bryan, G. L. 2006, ApJ, 641, 878

Tenorio-Tagle, G., \& Munoz-Tunon, C. 1998, MNRAS, 293, 299

Teyssier, R. 2002, A\&A, 385, 337

Thacker, R. J., \& Couchman, H. M. P. 2000, ApJ, 545, 728

Thacker, R. J., \& Couchman, H. M. P. 2001, ApJ, 555, L17

Thacker, R. J., Scannapieco, E., \& Davis, M. 2002, ApJ, 581, 836

Thornton, K., Gaudlitz, M., Janka, H.-T., \& Steinmetz, M. 1998, ApJ, 500, 95

Toomre, A. 1964, ApJ, 139, 1217

Veilleux, S., Cecil, G., \& Bland-Hawthorn, J. 2005, ARA\&A, 43, 769

Wada, K., Spaans, M., \& Kim, S. 2000, ApJ, 540, 797

Weil, M. L., Eke, V. R., \& Efstathiou, G. 1998, MNRAS, 300, 773

White, S. D. M., \& Frenk, C. S. 1991, ApJ, 379, 52

Yepes, G., Kates, R., Khokhlov, A., \& Klypin, A. 1997, MNRAS, 284, 235 